\title{
Mechanism of Levitation of a Slider with a Micro/Nanoscale Surface Structure on a Rotating Disk
}

\author{
Shigeru Yonemura $\cdot$ Susumu Isono $\cdot$ \\ Masashi Yamaguchi • Yoshiaki Kawagoe • \\ Takanori Takeno $\cdot$ Hiroyuki Miki $\cdot$ Toshiyuki Takagi
}

Received: 31 March 2014/ Accepted: 30 June 2014/Published online: 20 July 2014

(C) The Author(s) 2014. This article is published with open access at Springerlink.com

\begin{abstract}
It has been previously reported that the friction between a partially polished diamond-coated surface and a metal surface was drastically reduced to zero in the atmosphere as relative speed was increased (Nakamori et al. in Diam Relat Mater 14:2122-2126, 2005). On the other hand, it has also been reported that laser-textured surfaces have good tribological performance in the case of gas lubrication (Kligerman and Etsion in Tribol Trans $44: 472-478,2001)$. The surfaces in the aforementioned two cases have a micro/nanoscale structure. It is expected that both surfaces are levitated by a high-pressure gas film between sliding surfaces by the same mechanism. In the present work, the mechanism of high gas pressure generation is clarified by the performance of numerical simulations and by theoretical analysis. The following two features of pressure distributions on textured surfaces were found to induce high gas pressure. First, gas pressure increases in the direction of the counter surface's motion over the dimple region. Second, the pressure distribution over the flat region is convex upward, and hence, the high pressure obtained at the outlet of the dimple is maintained for a long distance in the flat region. The causes of such
\end{abstract}

S. Yonemura $(\bowtie) \cdot T$. Takagi

Institute of Fluid Science, Tohoku University, 2-1-1 Katahira,

Aoba-ku, Sendai 980-8577, Japan

e-mail: yonemura@ifs.tohoku.ac.jp

S. Isono $\cdot$ M. Yamaguchi $\cdot$ Y. Kawagoe $\cdot$ T. Takeno

Department of Nanomechanics, Graduate School of Engineering,

Tohoku University, 6-6-01 Aoba, Aramaki, Aoba-ku,

Sendai 980-8579, Japan

H. Miki

Frontier Research Institute for Interdisciplinary Sciences,

Tohoku University, 6-3 Aoba, Aramaki, Aoba-ku,

Sendai 980-8578, Japan pressure distributions are herein explained analytically. The governing factor of pressure distributions and the optimal dimple location in the period of the repeated surface pattern are also discussed. Furthermore, the knowledge obtained here is utilized to design the surface structure to obtain high gas pressure.

Keywords Surface texturing - Partially polished diamond coating · Molecular gas film lubrication - Direct simulation Monte Carlo method - High Knudsen number flow .

Rarefied gas dynamics

\section{Introduction}

Many studies on surface texturing have achieved improvements in tribological performance of sliding surfaces [1-7]. Surface texturing is expected to have three effects on tribological performance. The surface microstructures can act as a microhydrodynamic bearing by generating hydrodynamic pressure, as a microreservoir for a lubricant to prevent starved lubrication, or as a microtrap for wear debris [8]. In this work, the focus is only on the first role of surface texturing as a microhydrodynamic bearing.

In most studies of microhydrodynamic bearings, liquid is used as a lubricant. In cases where one surface moves relative to the other surface, the lubricant is drawn by the moving surface. Compared with the ambient pressure, the pressure becomes high over the region where the clearance converges and becomes low over the region where the clearance diverges. However, the pressure over the diverging region cannot be lower than the pressure at which cavities are formed in the liquid. Therefore, the pressure rise over the converging region is greater than the 
pressure drop over the diverging region, and then the pressure averaged over the whole surface becomes higher than the ambient pressure $[1,5]$.

As well as liquid lubrication, gas lubrication has also been studied for microhydrodynamic bearings. Kligerman and Etsion performed numerical simulations of gas lubrication for the case where two non-contacting cylindrical surfaces with microdimples rotate relative to each other [4]. They considered the surfaces, one of which had a regular network of microdimples. They investigated the optimal conditions to improve sealing performance by changing the values of parameters, e.g., seal clearance, dimple area density, and dimple aspect ratio. Other parametric analyses have been conduced to investigate optimal conditions of gas lubrication with surface texturing $[9,10]$. However, a clear understanding of the mechanism that induces high gas pressure over a textured surface would help us to design the optimal configuration of the surface texture without trial and error.

On the other hand, Nakamori et al. [11] found experimentally that the friction between a partially polished diamond-coated surface and a metal surface is drastically reduced to zero in the atmosphere at room temperature when its sliding speed becomes as much as $1 \mathrm{~m} / \mathrm{s}$. In their experiment, the mass of the slider with the diamond-coated surface was $280 \mathrm{~g}$ and the area of the sliding surface was approximately $1,960 \mathrm{~mm}^{2}$. Note that the sliding with low friction was noiseless in their experiment. This indicates that the slider was floating on the counter metallic surface, that is, the mechanism of this lubrication was not boundary lubrication, but rather gas film lubrication. More careful measurements of this low friction and noiseless sliding of partially polished diamond-coated surface were also reported by Miki et al. [12]. The scanning electron microscope (SEM) image of partially polished diamondcoated surface of Nakamori et al. [11] can be seen in Fig. 1 of [13]. They partially polished an as-deposited diamondcoated surface, and in consequence, the surface consisted of flat regions and hollow regions like valleys or dimples in micro/nanoscale. Yonemura et al. [14] applied the direct simulation Monte Carlo (DSMC) method to simulate this problem because the gas flow between two sliding surfaces is high Knudsen number flow. In their numerical simulations, they showed that the high gas pressure is induced by the dimple on the surface. But the mechanism of this generation of high gas pressure has not been explained.

High gas pressure is expected to be generated by the same mechanism both in the case of a partially polished diamond-coated surface and in the case of surface texturing. In the present work, in order to understand these phenomena clearly, the mechanism of high gas pressure generation induced by the micro/nanoscale surface

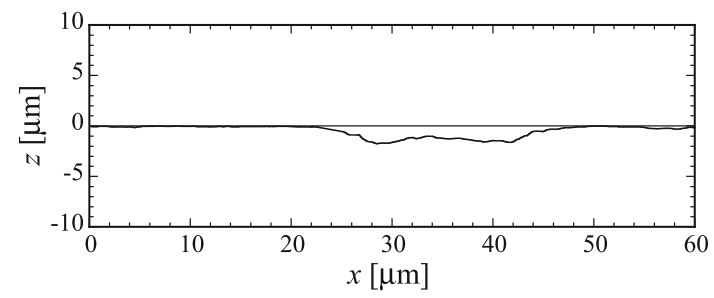

Fig. 1 Surface structure of partially polished diamond coating measured by atomic force microscope

structure was theoretically clarified with the assistance of numerical simulations. The knowledge obtained here will facilitate the design of the optimal surface structure to improve tribological performance.

\section{Numerical Method}

In the experiment of Nakamori et al. [11], as-deposited diamond-coated surfaces were polished until $R_{\mathrm{a}} \sim 0.5 \mu \mathrm{m}$. Small lumps of deposited ballas diamond were polished, the polished part forming flat regions, and the unpolished part forming valleys or dimples. Figure 1 shows an example of the surface structure on the partially polished diamond coating with the surface roughness $R_{\mathrm{a}}=0.34 \mu \mathrm{m}$.

In the case where the surface roughness is much smaller than the clearance between two sliding surfaces, the flow between parallel surfaces will become the Couette flow and will not generate high gas pressure. Therefore, the clearance must be on the order of surface roughness $R_{\mathrm{a}}$ or less. On the other hand, the mean free path $\lambda$ of atmospheric molecules is $0.065 \mu \mathrm{m}$. Therefore, the Knudsen number $K n(=\lambda / L)$ will be on the order of 0.1 or larger, where $L$ is the characteristic length of the flow. This indicates that the gas flow between two sliding surfaces cannot be treated as a continuum. Such kind of microscale gas flow is in nonequilibrium due to a lack of intermolecular collisions and is governed not by the Navier-Stokes equations, but by the Boltzmann equation.

Fukui and Kaneko derived the molecular gas film lubrication (MGL) equation [15] on the basis of the linearized Bhatnagar-Gross-Krook (BGK) model for the Boltzmann equation. This lubrication equation has often been used to analyze high Knudsen number lubrication problems [16-19]. On the other hand, the DSMC method [20] has been developed to analyze high Knudsen number flows like rarefied gas flows on the basis of the Boltzmann equation. This method has been applied to microscale gas flows in air-bearing slider problems [16-19, $21]$. Both the MGL equation and the DSMC method are herein used to analyze microscale gas flows between two sliding surfaces. 
Fig. 2 Computational domain

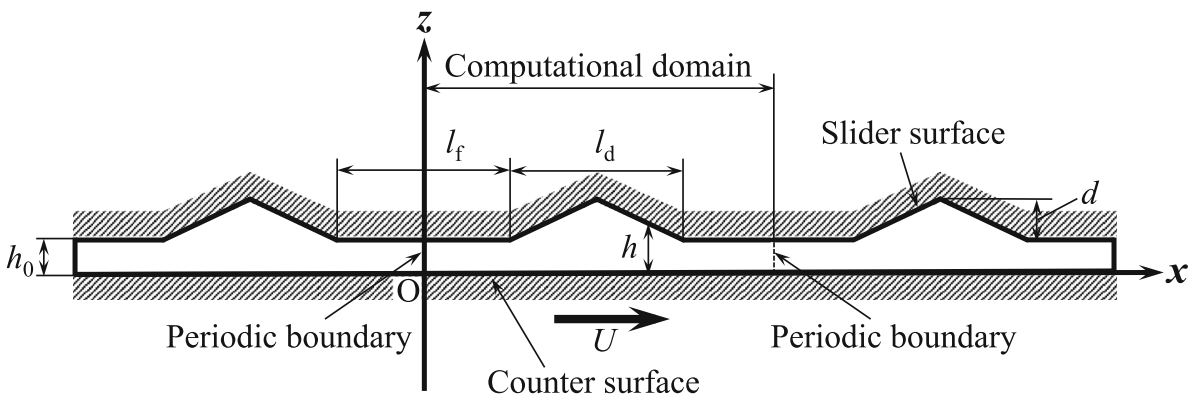

As shown in the SEM image [13] of the partially polished diamond-coated surface and Fig. 1, the surface consists of flat regions and shallow hollow regions like valleys or dimples. By reference to the surface structure of the partially polished diamond coating, a surface with an isosceles triangular dimple pattern shown in Fig. 2 is considered in the present work. This microscale dimple pattern is repeated periodically in the $x$ direction. The two-dimensional flow between the upper surface with dimples and the lower counter surface is analyzed by using the DSMC method and the MGL equation separately. The periodic boundary condition is applied for the inlet and the outlet of the computational domain in the $x$ direction. The lengths of the dimple region and the flat region of the upper surface are represented by $l_{\mathrm{d}}$ and $l_{\mathrm{f}}$, respectively. The total length $l_{\mathrm{T}}$ of the computational domain is given by $l_{\mathrm{T}}=l_{\mathrm{d}}+l_{\mathrm{f}}$. Dimple depth is denoted by $d$. The flat region of the upper surface is set to be parallel to the counter surface. The clearance between the two surfaces is denoted by $h$ and is set at its minimum value $h_{0}$ in the flat region. The counter surface moves at speed $U$ in the $x$ direction. The wall temperature is set at room temperature, $T_{0}=300 \mathrm{~K}$. The gas treated here is air. At the initial condition for DSMC simulations, the computational domain is filled with air molecules whose pressure and temperature are atmospheric pressure, $p_{\mathrm{a}}=1 \mathrm{~atm}=$ $101325 \mathrm{~Pa}$, and the room temperature, $T_{0}$, respectively. Therefore, the spatially averaged number density $n_{0}$ of air molecules is set at $n_{0}=2.4463 \times 10^{25} \mathrm{~m}^{-3}$ in DSMC simulations, where $n_{0}$ is given by the relation $p_{\mathrm{a}}=n_{0} k T_{0}$. In the present work, we consider only the steady gas flow between sliding surfaces. Therefore, the clearance $h_{0}$ is fixed during the execution of each DSMC simulation. As time passes, local gas pressure and temperature change although $n_{0}$ does not change, and eventually the gas flow reaches the steady state in DSMC simulations. In the MGL calculations, the steady state can be directly considered without advancing time.

\subsection{DSMC Simulation}

In the DSMC method, simulation molecules are samples of real molecules. One simulation molecule represents a large number of real molecules. Intermolecular collisions are treated stochastically on the basis of the collision probability in the DSMC cells [22]. Figure 3 shows the cell network for

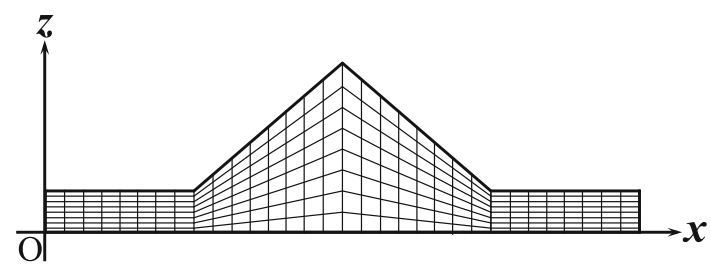

Fig. 3 Cell network for the DSMC simulation

the DSMC simulation. In the present DSMC simulations, the computational domain is divided into 800 equally partitioned cells in the $x$ direction and divided into 25 equally partitioned cells in the $z$ direction. The hard sphere molecular model is used for collision calculation. Air molecules are treated as hard spheres with diameters of $d_{\mathrm{m}}=0.37 \mathrm{~nm}$ and masses of $m=4.81 \times 10^{-26} \mathrm{~kg}$. The motions and collisions of molecules are traced, and hence, the time evolution of the gas flow field is simulated. After the flow has reached the steady state, the flow properties such as gas velocity, gas pressure, and gas temperature are sampled.

Since the mean free time of gas molecules is $1.4 \times 10^{-10} \mathrm{~s}$ in the atmosphere with room temperature, the time step $\Delta t$ for calculation of molecular motion and collision is set at $1.4 \times 10^{-11} \mathrm{~s}$. The velocities of molecules reflected on the wall are determined by using the diffuse reflection model with the wall temperature. The accommodation coefficient on the wall is set at unity. Intermolecular collisions are treated by using the maximum collision number method [22]. The flow field is treated as two-dimensional, but the molecular motions are treated as threedimensional. The slider surface receives impulses from incident gas molecules. The pressure distribution on the slider surface was obtained by integrating these impulses.

\subsection{MGL Equation}

To derive the lubrication equation, the following lubrication hypotheses are assumed.

(1) The thickness of the lubrication film is small compared with its length.

(2) The flow velocity in the thickness direction is negligibly small. 
(3) The gas density, the gas pressure, and the gas temperature are uniform in the thickness direction.

(4) The temperature on the wall is uniform and equal to the room temperature $T_{0}$ at any point.

From assumptions (1), (3), and (4), the gas temperature $T$ is considered to be uniform and equal to $T_{0}$ anywhere in the lubrication film because the lubrication film is very thin and surrounded by the isothermal wall with the room temperature $T_{0}$.

The continuity equation for steady flow of compressible fluid is as follows:

$\frac{\partial}{\partial x} \rho u+\frac{\partial}{\partial y} \rho v+\frac{\partial}{\partial z} \rho w=0$,

where $\rho$ is the gas density, $u, v$, and $w$ are the flow velocity components in the $x, y$, and $z$ directions, respectively. Since the flow is two-dimensional in the $x z$ plane, $v=0$. From assumption (2), $w=0$. The continuity equation (1) can be integrated from 0 to $h$ with respect to $z$ as follows:

$\frac{\mathrm{d}}{\mathrm{d} x} q=0$

where $q$ is given by

$q=\int_{0}^{h} \rho u \mathrm{~d} z$

Since the gas density $\rho$ depends on $x$ and not on $z$ on the basis of assumption (3), flow is not considered here. Therefore, the mass flow rate, $q$, can be expressed by the sum of those of the Poiseuille flow and the Couette flow as follows:

$q=q_{\mathrm{P}}+q_{\mathrm{C}}$

where $q_{\mathrm{P}}$ and $q_{\mathrm{C}}$ are the mass flow rates of the Poiseuille flow and the Couette flow, respectively.

The mass flow rate of the Couette flow is independent of the Knudsen number, $K n$, and is given by

$q_{\mathrm{C}}=\frac{1}{2} \rho U h$.

The mass flow rate of the Poiseuille flow for the continuum flow, i.e., $K n \ll 1$, can be given by

$q_{\mathrm{P}}=-\frac{\rho h^{3}}{12 \mu} \frac{\mathrm{d} p}{\mathrm{~d} x}$,

where $\mu$ is the viscosity, and $p$ is the gas pressure. The mass flow rate $q_{\mathrm{P}}$ depends on the Knudsen number and can be expressed as follows [15]:

$q_{\mathrm{P}}=-\frac{h^{2}}{\sqrt{2 R T_{0}}} Q_{\mathrm{P}}(D) \frac{\mathrm{d} p}{\mathrm{~d} x}$,

where $R$ is the ordinary gas constant, i.e., gas constant per unit mass, and is given by $k / m, k$ is the Boltzmann constant, $m$ is the molecular mass, $D$ is the inverse Knudsen number given by $D=\sqrt{\pi} /(2 K n)$, and $Q_{\mathrm{P}}(D)$ is the flow rate coefficient for the Poiseuille flow given as follows [23]:

$$
\begin{array}{ll}
Q_{\mathrm{P}}(D)=D / 6+1.0162+1.0653 / D-2.1354 / D^{2} & (5 \leq D), \\
Q_{\mathrm{P}}(D)=0.13852 D+1.25087+0.15653 / D-0.00969 / D^{2} & (0.15 \leq D<5), \\
Q_{\mathrm{P}}(D)=-2.22919 D+2.10673+0.01653 / D-0.0000694 / D^{2} & (0.01 \leq D<0.15) .
\end{array}
$$

$$
q=\rho \int_{0}^{h} u \mathrm{~d} z
$$

More specifically, $q$ represents the mass flow rate in the $x$ direction between surfaces in the case where the width of the flow field in the $y$ direction is considered as unity. In the case of usual continuum flows, the solution of the NavierStokes equations can be given by a combination of the Couette flow induced by the moving wall and the Poiseuille flow induced by the pressure gradient under assumptions (2) and (3). Even in the case of a high Knudsen number region, the flows in the lubricated film can be expressed as a combination of the Couette flow and the Poiseuille flow [15]. From assumption (4), the effect of thermal creep
In the case of $K n \ll 1$, i.e., $D \gg 1, Q_{\mathrm{P}}(D)$ can be given by $Q_{\mathrm{P}}(D)=D / 6$, and then Eq. (8) results in Eq. (7). By substituting Eqs. (5), (6), and (8) into Eq. (2), the following molecular gas film lubrication (MGL) equation [15] is obtained:

$\frac{\mathrm{d}}{\mathrm{d} x}\left[\frac{h^{2}}{\sqrt{2 R T_{0}}} Q_{\mathrm{P}}(D) \frac{\mathrm{d} p}{\mathrm{~d} x}-\frac{1}{2} \rho U h\right]=0$.

The spatially averaged gas pressure $\bar{p}_{\text {space }}$ in the computational domain is set at the atmospheric pressure $p_{\mathrm{a}}$ for the present MGL calculations except them in Sect. 3.6. The gas temperature at any point in the lubrication film is also assumed to be equal to the room temperature $T_{0}$ as mentioned above. Therefore, this setting of the spatially averaged gas pressure for the MGL calculation corresponds to 
Fig. 4 Distributions of a gas flow velocity, $\mathbf{b}$ gas pressure, $\mathbf{c}$ molecular number density, and d gas temperature obtained in the DSMC simulation.

$\left(l_{\mathrm{d}}=l_{\mathrm{f}}=23.04 \mu \mathrm{m}\right.$,

$d=1.44 \mu \mathrm{m}, U=10 \mathrm{~m} / \mathrm{s}$,

$\left.h_{0}=0.14 \mu \mathrm{m}\right)$
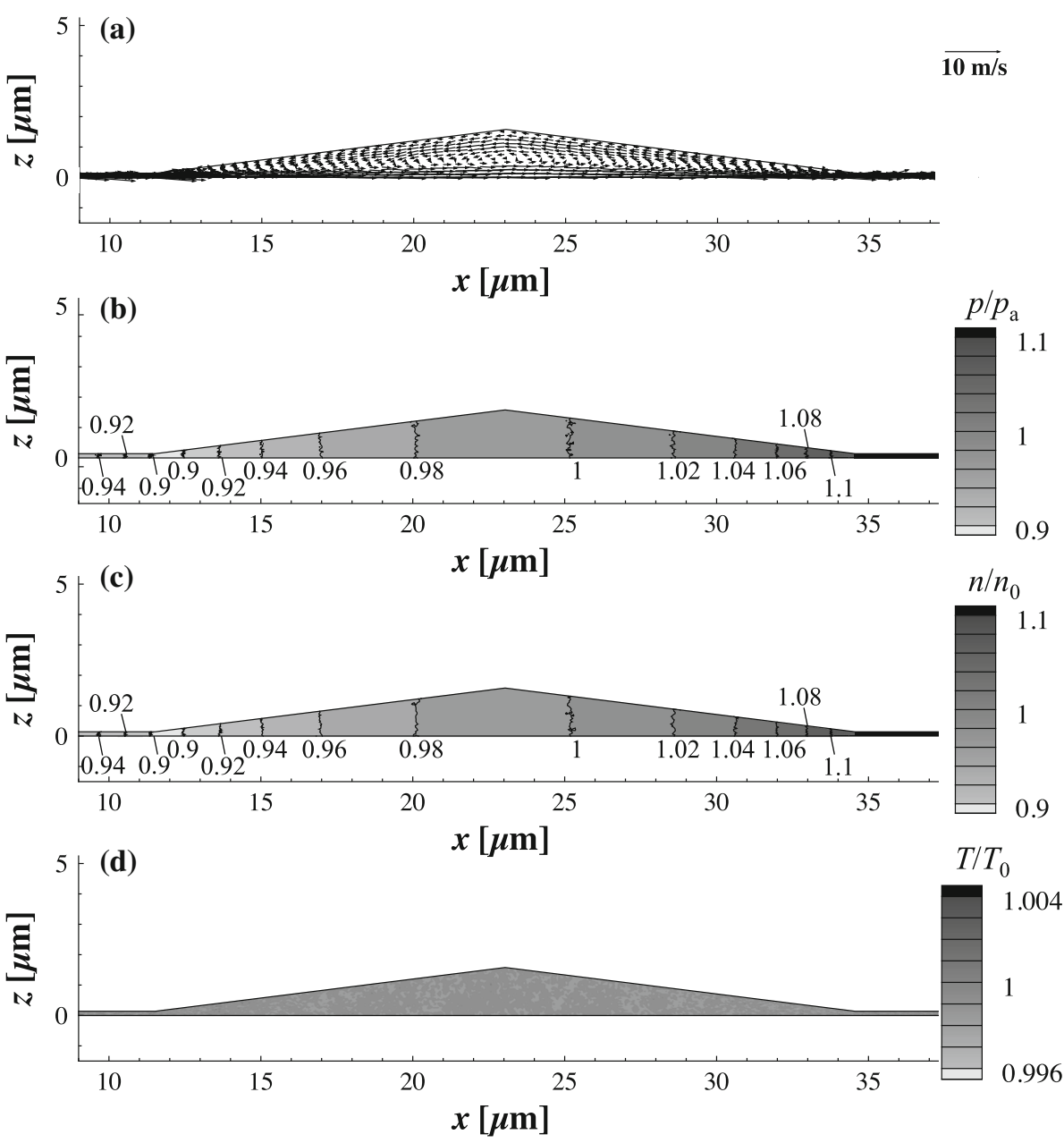

the setting of the spatially averaged number density $n_{0}$ for the DSMC simulation.

The MGL equation is solved by using the following boundary condition:

$p(0)=p\left(l_{\mathrm{T}}\right)=p_{\mathrm{bc}}$,

where $p_{\mathrm{bc}}$ is a tentative boundary condition of the gas pressure. The MGL equation is solved by using the finite difference method with this tentative boundary condition. The number of grid points in the computational domain is 10000. After the MGL equation has been solved by using this tentative boundary condition, the spatially averaged gas pressure $\bar{p}_{\text {space }}$ in the computational domain is calculated by

$\bar{p}_{\text {space }}=\frac{\int_{0}^{l_{\mathrm{T}}} p h \mathrm{~d} x}{\int_{0}^{l_{\mathrm{T}}} h \mathrm{~d} x}$,

and the boundary condition is renewed as $p_{\mathrm{bc}}=p_{\mathrm{bc}} \times$ $p_{\mathrm{a}} / \bar{p}_{\text {space }}$, and then the MGL equation is solved again. These processes are repeated until the spatially averaged pressure $\bar{p}_{\text {space }}$ becomes equal to $p_{\mathrm{a}}$. The boundary condition for the MGL calculations in Sect. 3.6 will be described there.
The viscosity $\mu$ for the MGL calculations is set to be equal to that obtained from the settings for the DSMC simulations by using

$\mu=\frac{1}{2} \rho_{0} \bar{C} \lambda_{0}$

where the density $\rho_{0}$ is given by $\rho_{0}=m n_{0}$, the average peculiar velocity $\bar{C}$ of gas molecules is given by $\bar{C}=\sqrt{8 k T_{0} / \pi m}$, the characteristic mean free path $\lambda_{0}$ is given by $\lambda_{0}=1 /\left(\sqrt{2} n_{0} \sigma_{\mathrm{T}}\right)$, and the total collision cross section $\sigma_{\mathrm{T}}$ of hard sphere gas molecules is given by $\sigma_{\mathrm{T}}=\pi d_{\mathrm{m}}^{2}$. In the MGL calculations, the viscosity $\mu$ is set at $\mu=1.85 \times 10^{-5} \mathrm{~Pa} \cdot \mathrm{s}$, which is estimated from the settings for the DSMC simulations, i.e., the molecular diameter $d_{\mathrm{m}}=0.37 \mathrm{~nm}$, the molecular mass $m=4.81 \times 10^{-26} \mathrm{~kg}$, the reference gas temperature $T_{0}=300 \mathrm{~K}$, and the molecular number density $n_{0}=2.4463 \times 10^{25} \mathrm{~m}^{-3}$. The characteristic mean free path $\lambda_{0}$ is estimated as $0.0672 \mu \mathrm{m}$ for these conditions.

The local inverse Knudsen number $D$ for the MGL calculations is given by using the local Knudsen number $K n$ as $D=\sqrt{\pi} /(2 K n)$, where $K n$ is given by $K n=\lambda / h$, the 
local mean free path $\lambda$ is given by $\lambda=1 /\left(\sqrt{2} n \sigma_{\mathrm{T}}\right)$, and the local molecular number density $n$ is given by $n=p / k T_{0}$.

\section{Results and Discussion}

\subsection{Typical Flow Between Two Sliding Surfaces}

Figure 4 shows typical distributions of the gas flow velocity, the gas pressure $p$, the molecular number density $n$, and the gas temperature $T$ obtained in the DSMC simulation for the case of $l_{\mathrm{d}}=l_{\mathrm{f}}=23.04 \mu \mathrm{m}, d=1.44 \mu \mathrm{m}$, $U=10 \mathrm{~m} / \mathrm{s}$, and $h_{0}=0.14 \mu \mathrm{m}$, respectively. The rightward gas flow is induced by the rightward motion of the lower counter surface. However, the major portion of the gas flow induced in the dimple region cannot pass through the narrow channel in the flat region and is repelled at the converging part of the dimple. Therefore, a vortical flow appears in the dimple. Since some portion of gas flow can pass through the channel, the net gas flow at any $x$ is rightward. A similar vortex appears in the cases where the minimum clearance $h_{0}$ is small. Such a kind of vortex will disappear in the case where the minimum clearance $h_{0}$ is not so small compared with the dimple depth $d$. The gas pressure drops at the left edge of the dimple and rises at the right edge of the dimple, i.e., the gas pressure increases in the $x$ direction over the dimple region. The gas temperature is uniform in the computational domain as we assumed for the MGL calculations in Sect. 2.2. This is because the gas flow channel is very narrow and surrounded by isothermal solid surfaces. Because of uniform temperature, the molecular number density distribution becomes similar to the gas pressure distribution, as shown in Fig. 4.

Figure 5 shows the distribution of the gas pressure exerted on the bottom surface of the slider for various sliding speeds $U$ under the condition of $l_{\mathrm{d}}=l_{\mathrm{f}}=23.04 \mu \mathrm{m}$, $d=1.44 \mu \mathrm{m}$, and $h_{0}=0.14 \mu \mathrm{m}$. The line figure in the lower half of Fig. 5 shows the shape of the lubricated region. The arrow pointing right indicates the direction of the motion of the lower counter surface with speed $U$. Note that the shape of the lubricated region is enlarged in the $z$ direction. The pressure distributions obtained in the MGL calculations coincide well with those obtained in the DSMC simulations in the cases considered here. These agreements show the validity of the DSMC simulation and that of the MGL calculation in the present study.

In the dimple region, the gas pressure $p$ monotonically increases in the $x$ direction, i.e., in the direction of the motion of the lower counter surface, or in the direction of the net gas flow. The gas pressure reaches its minimum at the diverging edge of the triangular dimple, i.e., at the inlet of the dimple, and reaches its maximum at the converging

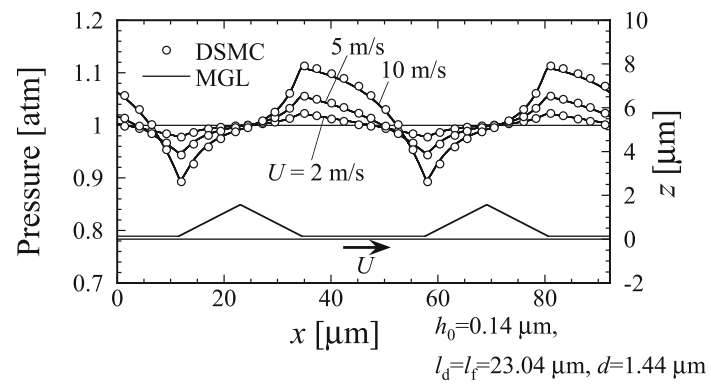

Fig. 5 Pressure distributions obtained in the DSMC simulations and the MGL calculations for various sliding speeds $U$. $\left(l_{\mathrm{d}}=l_{\mathrm{f}}=23.04 \mu \mathrm{m}\right.$, $\left.d=1.44 \mu \mathrm{m}, h_{0}=0.14 \mu \mathrm{m}\right)$

edge of the dimple, i.e., at the outlet of the dimple. Note that the left and right edges of the dimple are referred to as "inlet" and "outlet" of the dimple, respectively, with reference to the direction of the net gas flow, i.e., with reference to the direction of the counter surface's motion. Similarly, the left and right edges of the flat region are referred to as "inlet" and "outlet" of the flat region, respectively. The gas pressure over the diverging region of the dimple is less than the atmospheric pressure $p_{\mathrm{a}}$, while that over the converging region of the dimple is larger than $p_{\mathrm{a}}$. Look at the pressure distribution for the case of $U=10 \mathrm{~m} / \mathrm{s}$ shown in Fig. 5. Note that this case is the same as the case shown in Fig. 4. The pressure rise over the converging region is canceled by the pressure drop over the diverging region, and hence, the pressure averaged over the dimple region is almost equal to $p_{\mathrm{a}}$. On the other hand, the gas pressure over the flat region monotonically decreases in the $x$ direction. The gradient of the pressure distribution at the left edge of the flat region, i.e., at the inlet of the flat region, is gentle, while that at the right edge of the flat region, i.e., at the outlet of the flat region, is steep. Consequently, the pressure distribution over the flat region is convex upward, and this shape of the pressure distribution causes the generation of high gas pressure.

Since the atmospheric pressure $p_{\mathrm{a}}(=1 \mathrm{~atm})$ is exerted on the top surface of the slider, the lift force can be given by the pressure difference $\bar{p}_{\text {surf }}-p_{\mathrm{a}}$ between the bottom and top surfaces of the slider, where $\bar{p}_{\text {surf }}$ is the gas pressure averaged over the whole bottom surface of the slider. Figure 6 shows the effect of the sliding speed $U$ on the gas pressure $\bar{p}_{\text {surf }}$. These pressures are obtained by the MGL calculations. As $U$ increases, the average gas pressure $\bar{p}_{\text {surf }}$ increases because the increase of pressure in the $x$ direction over the dimple region becomes stronger and the pressure distribution in the flat region changes from linear to convex upward, as shown in Fig. 5 . In the case of $U=10 \mathrm{~m} / \mathrm{s}$, the superficially averaged gas pressure $\bar{p}_{\text {surf }}$ is $1.019 \mathrm{~atm}$, although the spatially averaged gas pressure $\bar{p}_{\text {space }}$ is still equal to $p_{\mathrm{a}}$. The lift force $\bar{p}_{\text {surf }}-p_{\mathrm{a}}$ is about $1,900 \mathrm{~Pa}$ in 


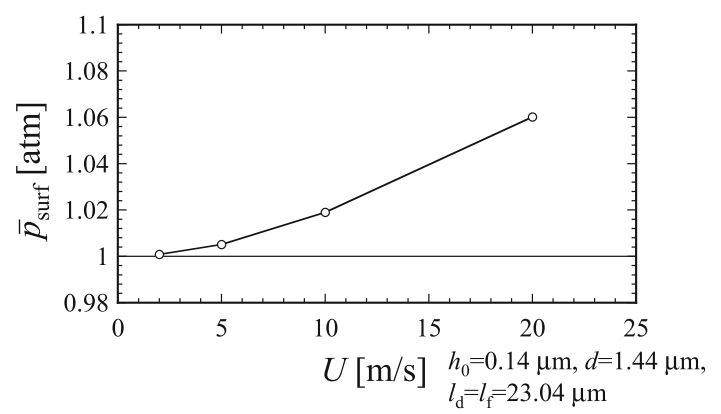

Fig. 6 Effect of the sliding speed $U$ on superficially averaged gas pressure $\bar{p}_{\text {surf }} \cdot\left(l_{\mathrm{d}}=l_{\mathrm{f}}=23.04 \mu \mathrm{m}, d=1.44 \mu \mathrm{m}, h_{0}=0.14 \mu \mathrm{m}\right)$

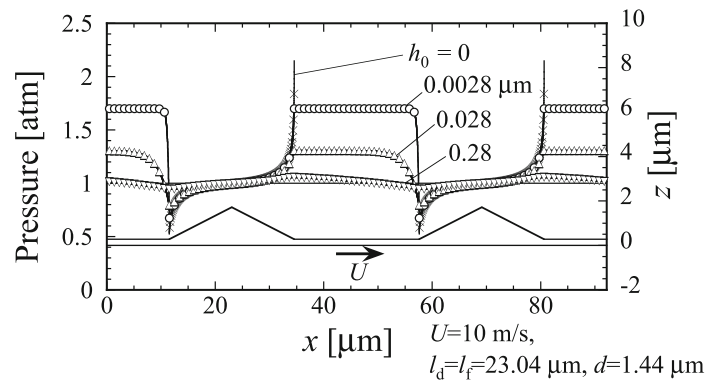

Fig. 7 Effect of the clearance $h_{0}$ on the pressure distribution. $\left(l_{\mathrm{d}}=l_{\mathrm{f}}=23.04 \mu \mathrm{m}, d=1.44 \mu \mathrm{m}, U=10 \mathrm{~m} / \mathrm{s}\right)$

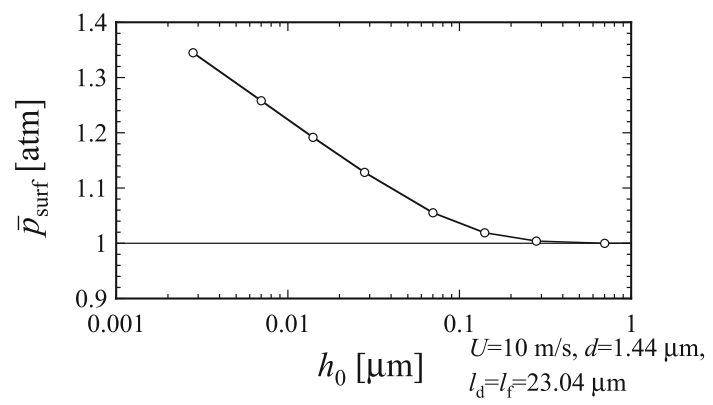

Fig. 8 Effect of the clearance $h_{0}$ on superficially averaged gas pressure $\bar{p}_{\text {surf }} .\left(l_{\mathrm{d}}=l_{\mathrm{f}}=23.04 \mu \mathrm{m}, d=1.44 \mu \mathrm{m}, U=10 \mathrm{~m} / \mathrm{s}\right)$

this case. This is sufficiently large to levitate the slider of $280 \mathrm{~g}$ with the sliding surface $1,960 \mathrm{~mm}^{2}$ used in the experiment of Nakamori et al. [11].

\subsection{Mechanism of High Gas Pressure Generation}

Not only in the case of gas lubrication, but also in the case of liquid lubrication using surface texturing, the pressure rises in the converging region of the dimple and drops in the diverging region of the dimple. In the case of liquid, however, the pressure over the diverging region of the dimple cannot be lower than the liquid vapor pressure due to generation of cavities. In consequence, the pressure drop over the diverging region of the dimple is limited, and hence, the pressure rise over the converging region of the dimple is greater. This asymmetric pressure distribution over the dimple region makes the average gas pressure greater than the ambient atmospheric pressure $[1,5]$.

As well as liquid lubrication, in the case of gas lubrication using surface texturing, the asymmetric pressure distribution in which the pressure rise over the converging region of the dimple is greater than the pressure drop over the diverging region of the dimple also appears in the dimple region under some conditions. See Fig. 7, which shows pressure distributions for a variety of minimum clearances $h_{0}$ obtained by the MGL calculations. From this point on, all results are obtained by the MGL calculations since the validity of our MGL calculation was verified, and as its computational load is much smaller than the DSMC simulation. The line figure in the lower half of Fig. 7 shows the shape of the lubricated region for the case of $h_{0}=0.28 \mu \mathrm{m}$. As the clearance $h_{0}$ decreases from 0.28 to $0.028 \mu \mathrm{m}, 0.0028 \mu \mathrm{m}$, or 0 , the pressure rise over the converging region of the dimple and the pressure drop over the diverging region become intense, and the pressure rise becomes greater than the pressure drop. In previous theoretical studies $[9,10]$, the generation of high average gas pressure has been explained as resulting from this asymmetric pressure distribution over the dimple region in the case of gas lubrication using surface texturing as well as in the case of liquid lubrication.

However, in the case of $U=10 \mathrm{~m} / \mathrm{s}$ shown in Fig. 5, gas pressure sufficiently high to levitate the slider of Nakamori et al. is obtained in spite of the symmetric pressure distribution over the dimple region. In this case, since the pressure distribution over the dimple region is symmetric, the gas pressure averaged over the dimple region is almost equal to the ambient pressure $p_{\mathrm{a}}$, as mentioned in Sect. 3.1. Therefore, the pressure distribution over the dimple region does not make a direct contribution to the lift force, whereas the gas pressure $\bar{p}_{\text {surf }}$ averaged over the whole bottom surface of the slider is higher than the ambient pressure $p_{\mathrm{a}}$ because the pressure distribution over the flat region is convex upward. This indicates that the asymmetric pressure distribution over the dimple region is not always required to obtain high average gas pressure. Even in the case where the pressure distribution over the dimple region is symmetric, a pressure difference is produced between the inlet and the outlet of the flat region by the presence of the dimple, and then this pressure difference is used to advantage to make the average gas pressure over the whole surface higher with the help of the convex-upward pressure distribution over the flat region. The asymmetric pressure distribution over the dimple region is beneficial to obtain higher average gas pressure as shown in the cases of small $h_{0}$ 's in Fig. 7 under the condition that the spatially averaged pressure $\bar{p}_{\text {space }}$ is equal to the ambient 
pressure $p_{\mathrm{a}}$. However, the asymmetric pressure distribution in the dimple region is not always beneficial, as we will discuss later in the last paragraph of Sect. 3.6.

Look at the pressure distributions for the cases of $h_{0}=0.028 \mu \mathrm{m}$ and $h_{0}=0.0028 \mu \mathrm{m}$ in Fig. 7. The gradients of these pressure distributions at the inlet of the flat region are 0 . Therefore, the maximum pressure obtained at the outlet of the dimple region is maintained for a long distance in the flat region. Finally, the gas pressure suddenly drops to the minimum at the inlet of the next dimple. Such a pressure distribution in the flat region, which is like a plateau, is beneficial to increase the average gas pressure.

From these results, it is found that the following two features of a pressure distribution play a key role in obtaining high average gas pressure.

(1) In the dimple region, gas pressure should increase in the direction of the counter surface's motion. This increase of gas pressure is induced by the presence of the dimple.

(2) The pressure distribution over the flat region should be convex upward. The combination of a gentler gradient at the inlet of the flat region and a steeper gradient at the outlet of the flat region is more beneficial for increasing the gas pressure averaged over the flat region.

Figure 8 shows the effect of the minimum clearance $h_{0}$ on the gas pressure $\bar{p}_{\text {surf }}$ averaged over the whole bottom surface of the slider. As $h_{0}$ decreases, the average gas pressure $\bar{p}_{\text {surf }}$ increases because the foregoing two features and an asymmetric pressure distribution over the dimple region become more remarkable. On the other hand, the strong gas pressure disappears when the minimum clearance $h_{0}$ comes close to the order of the dimple depth $d(=1.44 \mu \mathrm{m})$. This result reinforces our expectation that the clearance should be on the order of the surface roughness $R_{\mathrm{a}}$ or less for high gas pressure generation. The reason for the increase of $\bar{p}_{\text {surf }}$ with increasing $U$ shown in Fig. 6 is also because the two foregoing features become stronger as $U$ increases, as shown in Fig. 5. In the following subsections, let us consider the causes of the foregoing two features of pressure distributions.

\subsection{Cause of Feature (1) of Pressure Distributions}

First, let us consider the reason why the gas pressure increases over the dimple region in the direction of the counter surface's motion, i.e., the cause of feature (1) of pressure distributions. Consideration of the case of $h_{0}=0$ shown in Fig. 7 is helpful for understanding this reason. In this case, the net gas flow rate is 0 , i.e., $q=q_{\mathrm{P}}+q_{\mathrm{C}}=0$. By substituting Eqs. (6) and (8) into this equation, we have

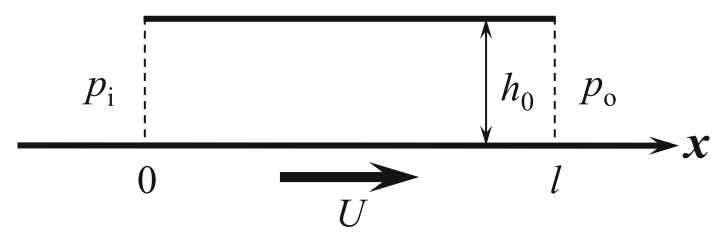

Fig. 9 Sliding of two parallel plates

$\frac{h^{2}}{\sqrt{2 R T_{0}}} Q_{\mathrm{P}}(D) \frac{\mathrm{d} p}{\mathrm{~d} x}-\frac{p U h}{2 R T_{0}}=0$,

where the relation $p=\rho R T_{0}$ is used. Since the flow field is not periodic in this case, this equation was solved not by using the boundary condition (11) but by using the condition that the spatially averaged gas pressure $\bar{p}_{\text {space }}$ is equal to the ambient atmospheric pressure $p_{\mathrm{a}}$, to obtain the pressure distribution for $h_{0}=0$ shown in Fig. 7. From Eq. (14), we have

$\frac{\mathrm{d} p}{\mathrm{~d} x}=\frac{p U}{h Q_{\mathrm{P}}(D) \sqrt{2 R T_{0}}}$.

This equation indicates that the gradient $\mathrm{d} p / \mathrm{d} x$ is positive at any point in the dimple in the case of $h_{0}=0$ because all factors in the right-hand side of Eq. (15) are positive. Therefore, the pressure monotonically increases over the dimple region as shown in the case of $h_{0}=0$ in Fig. 7. The case of very small clearance like $h_{0}=0.0028 \mu \mathrm{m}$ can be regarded as a situation where gas is leaking through the narrow clearance from the outlet of the dimple to the inlet of the next dimple in the case where both surfaces are almost in contact. The spatial increase of gas pressure over the dimple region in the cases of small clearance can be understood as well as in the case of $h_{0}=0$. In the case of $h_{0}=0.0028 \mu \mathrm{m}$, the minimum pressure at the inlet of the dimple and the maximum pressure at the outlet of the dimple are relieved to some extent from those in the case of $h_{0}=0$ because of gas leakage. As the clearance $h_{0}$ increases, the gas leakage becomes larger and the pressure increase over the dimple becomes smaller, as shown in Fig. 7.

From Eq. (15), the positive pressure gradient $\mathrm{d} p / \mathrm{d} x$ increases in proportion to the sliding speed $U$. Therefore, the pressure increase over the dimple region in the direction of the counter surface's motion, i.e., feature (1), becomes stronger as the sliding speed $U$ increases, as shown in Fig. 5.

\subsection{Cause of Feature (2) of Pressure Distributions}

Next, in order to understand feature (2) of pressure distributions seen in the flat region, let us consider the sliding of the two parallel plates shown in Fig. 9. The left edge and 


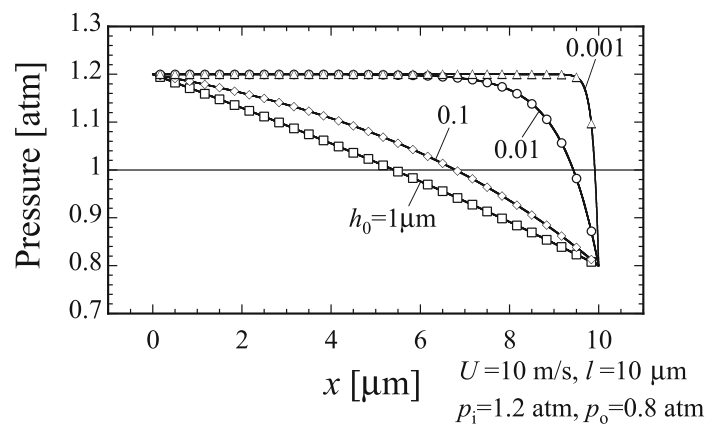

Fig. 10 Effect of the clearance $h_{0}$ on the pressure distribution in the case of the sliding of two parallel plates. $(U=10 \mathrm{~m} / \mathrm{s}, l=10 \mu \mathrm{m}$, $\left.p_{\mathrm{i}}=1.2 \mathrm{~atm}, p_{\mathrm{o}}=0.8 \mathrm{~atm}\right)$

the right edge of the lubricated region are an inlet and an outlet, respectively. The pressures $p_{\mathrm{i}}$ and $p_{\mathrm{o}}$ are those at the inlet and the outlet, respectively. Here, only the cases of $p_{\mathrm{i}}>p_{\mathrm{o}}$ are considered. The pressure distributions obtained by the MGL calculations for various clearances $h_{0}$ in the case where $p_{\mathrm{i}}=1.2 \mathrm{~atm}, p_{\mathrm{o}}=0.8 \mathrm{~atm}, l=10 \mu \mathrm{m}$, and $U=10 \mathrm{~m} / \mathrm{s}$ are shown in Fig. 10, where $l$ is the length of the lubricated region. In the cases considered in Fig. 10, the pressure distributions show monotonic decreases of the gas pressure $p$ and the pressure gradient $\mathrm{d} p / \mathrm{d} x(<0)$ in the $x$ direction, i.e., these distributions are convex upward. As $h_{0}$ decreases, the pressure gradient at the inlet becomes gentler and that at the outlet becomes steeper, and hence, the average gas pressure becomes high. In the case of a sufficiently small $h_{0}$, the pressure $p$ is asymptotic to its maximum value $p_{\mathrm{i}}$ for $x \rightarrow 0$ and the average gas pressure becomes close to the maximum pressure $p_{\mathrm{i}}$.

Since the net gas flow rate is constant at any point in the lubricated region, the Poiseuille flow rate $q_{\mathrm{P}}$ and the Couette flow rate $q_{\mathrm{C}}$ satisfy the relation,

$q_{\mathrm{Pi}}+q_{\mathrm{Ci}}=q_{\mathrm{Po}}+q_{\mathrm{Co}}$,

where the subscripts " $\mathrm{i}$ " and "o" represent the values at the "inlet" and the "outlet," respectively. Immediately, we have

$q_{\mathrm{Po}}-q_{\mathrm{Pi}}=q_{\mathrm{Ci}}-q_{\mathrm{Co}}$.

This indicates that the decrease of the flow rate $q_{\mathrm{C}}$ of the Couette flow along the channel is compensated by the increase of the flow rate $q_{\mathrm{P}}$ of the Poiseuille flow. From Eqs. (6), (8), (17), $h=h_{0}$, and $p=\rho R T_{0}$, we have

$$
\left[Q_{\mathrm{P}}(D)\left(-\frac{\mathrm{d} p}{\mathrm{~d} x}\right)\right]_{\mathrm{o}}-\left[Q_{\mathrm{P}}(D)\left(-\frac{\mathrm{d} p}{\mathrm{~d} x}\right)\right]_{\mathrm{i}}=\frac{U\left(p_{\mathrm{i}}-p_{\mathrm{o}}\right)}{h_{0} \sqrt{2 R T_{0}}} .
$$

As the clearance $h_{0}$ decreases, the magnitude of the righthand side of Eq. (18) increases in inverse proportion to $h_{0}$. The decrease of $h_{0}$ also induces a decrease of the inverse
Knudsen number $D$, and hence, it changes the flow rate coefficient $Q_{\mathrm{P}}(D)$. However, the change of $Q_{\mathrm{P}}(D)$ is small compared with the increase of the RHS of Eq. (18) because $Q_{\mathrm{P}}(D)$ changes only slightly against $D$ for the range of $D$ considered here. The range of values of $Q_{\mathrm{P}}(D)$ is $1.539<Q_{\mathrm{P}}(D)<3.060$ for $0.01<D<10$ [23]. On the other hand, although the inverse Knudsen number $D$ changes along the parallel channel in proportion to the pressure $p$, the spatial change of $Q_{\mathrm{P}}(D)$ along the channel is also small for the same reason. Therefore, the increase of the RHS of Eq. (18) increases the difference of the pressure gradient $(-\mathrm{d} p / \mathrm{d} x)$ between the inlet and the outlet. Hence, as the clearance $h_{0}$ decreases, the gradient of the pressure distribution at the outlet becomes steeper and that at the inlet becomes gentler. This is because the Couette flow rate is in proportion to $h_{0}$, whereas the Poiseuille flow rate is in proportion to about $h_{0}^{2}$ to $h_{0}^{3}$. [See Eqs. (6), (7), and (8)]. Therefore, a steeper pressure gradient $(-\mathrm{d} p / \mathrm{d} x)$ at the outlet is required for smaller $h_{0}$ in order to compensate the decrease of the Couette flow, i.e., $q_{\mathrm{Ci}}-q_{\mathrm{Co}}=\left(p_{\mathrm{i}}-p_{\mathrm{o}}\right)$ $U h_{0} / 2 R T$. As a result, the pressure distribution becomes more convex upward and higher average gas pressure is obtained.

The increase of $U$ also increases the RHS of Eq. (18). Therefore, as the sliding speed $U$ increases, the pressure gradient $(-\mathrm{d} p / \mathrm{d} x)$ at the outlet becomes steeper and that at the inlet becomes gentler, i.e., feature (2) becomes stronger, as shown in Fig. 5.

Although the discussion in the previous paragraphs is useful to understand the reason for the gentle pressure gradient at the inlet and the steep pressure gradient at the outlet for small $h_{0}$ or for large $U$, it is rather rough because we did not consider the change of $Q_{\mathrm{P}}(D)$. Next let us discuss feature (2) of pressure distributions precisely by considering the effect of the change of $Q_{\mathrm{P}}(D)$ against $D$. To generalize this problem, let us use the non-dimensionalized form of Eq. (10). Using the non-dimensional quantities given by

$X=\frac{x}{l}, \quad H=\frac{h}{h_{0}}, \quad P=\frac{p}{p_{\mathrm{a}}}$,

Eq. (10) is non-dimensionalized as

$\frac{\mathrm{d}}{\mathrm{d} X}\left[Q_{\mathrm{P}}(D) \frac{6 H^{2}}{D_{0}} \frac{\mathrm{d} P}{\mathrm{~d} X}-\Lambda P H\right]=0$,

where $D_{0}$ is the characteristic inverse Knudsen number defined by the ambient atmospheric pressure $p_{\mathrm{a}}$ and the minimum clearance $h_{0}$ as $D_{0}=\sqrt{\pi} /\left(2 K n_{0}\right)=p_{\mathrm{a}} h_{0} /$ $\mu \sqrt{2 R T_{0}}, K n_{0}$ is the characteristic Knudsen number defined by $p_{\mathrm{a}}, T_{0}$ and $h_{0}$, and $\Lambda$ is the bearing number defined by $\Lambda=6 \mu U l / p_{\mathrm{a}} h_{0}^{2}$. The inverse Knudsen number $D$ is expressed by using $D_{0}$ as $D=D_{0} P H$. Since $H=1$ for the problem of sliding of two parallel plates, we have 


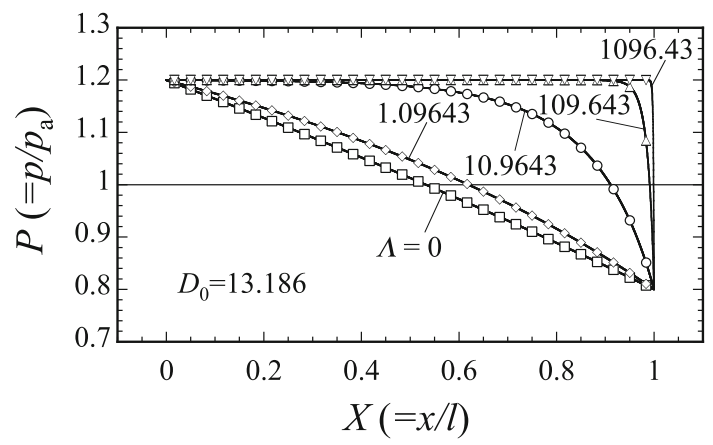

Fig. 11 Effect of the bearing number $\Lambda$ on the pressure distribution in the case of the sliding of two parallel plates. $\left(D_{0}=13.186, P_{\mathrm{i}}=1.2\right.$, $\left.P_{\mathrm{o}}=0.8\right)$

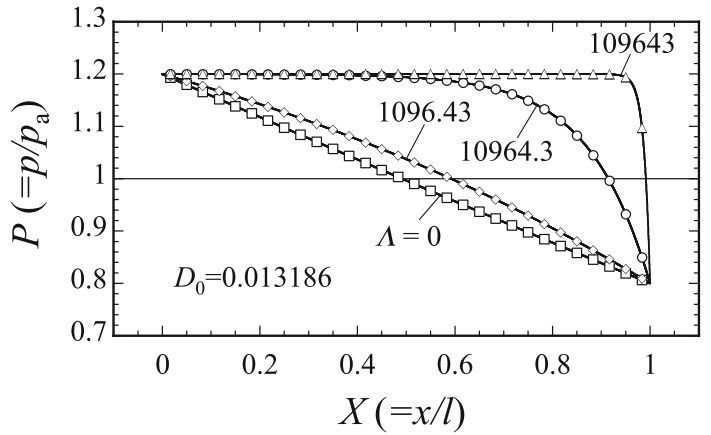

Fig. 12 Effect of the bearing number $\Lambda$ on the pressure distribution in the case of the sliding of two parallel plates. $\left(D_{0}=0.013186\right.$, $\left.P_{\mathrm{i}}=1.2, P_{\mathrm{o}}=0.8\right)$

$\frac{\mathrm{d}^{2} P}{\mathrm{~d} X^{2}}=\frac{D_{0}}{6 Q_{\mathrm{P}}(D)}\left[-6 \frac{\mathrm{d} Q_{\mathrm{P}}(D)}{\mathrm{d} D}\left(\frac{\mathrm{d} P}{\mathrm{~d} X}\right)+\Lambda\right] \frac{\mathrm{d} P}{\mathrm{~d} X}$,

where $\mathrm{d} Q_{\mathrm{P}}(D) / \mathrm{d} X=\left(\mathrm{d} Q_{\mathrm{P}}(D) / \mathrm{d} D\right)(\mathrm{d} D / \mathrm{d} P)(\mathrm{d} P / \mathrm{d} X)$ and $\mathrm{d} D / \mathrm{d} P=\mathrm{d}\left(D_{0} P H\right) / \mathrm{d} P=\mathrm{d}\left(D_{0} P\right) / \mathrm{d} P=D_{0}$ are used. The gradient $\mathrm{d} Q_{\mathrm{P}}(D) / \mathrm{d} D$ is positive for $D>1$ and negative for $D<1$ [23]. Since the pressure $P_{\mathrm{i}}\left(=p_{\mathrm{i}} / p_{\mathrm{a}}\right)$ at the inlet $X=0$ is larger than the pressure $P_{\mathrm{o}}\left(=p_{\mathrm{o}} / p_{\mathrm{a}}\right)$ at the outlet $X=1$, there is one point, $X=c(0<c<1)$, at which $\mathrm{d} P / \mathrm{d} X$ is negative. Whether $\mathrm{d}^{2} P / \mathrm{d} X^{2}$ is negative or positive at the point $X=c$ depends on values of $\mathrm{d} Q_{\mathrm{P}}(D) / \mathrm{d} D$, i.e., $D$, and the bearing number $\Lambda$.

In the case of $D>1$ at any point in the lubricated region, $\mathrm{d} Q_{\mathrm{P}}(D) / \mathrm{d} D$ is positive, and hence, not only $\mathrm{d} P / \mathrm{d} X$ but also $\mathrm{d}^{2} P / \mathrm{d} X^{2}$ is negative at the point $X=c$ since the characteristic inverse Knudsen number $D_{0}$, the flow rate coefficient $Q_{\mathrm{P}}(D)$, and the bearing number $\Lambda$ are not less than 0 by their definitions. For $X>c, \mathrm{~d} P / \mathrm{d} X(<0)$ decreases in the $X$ direction because $\mathrm{d}^{2} P / \mathrm{d} X^{2}<0$, and Eq. (21) indicates that $\mathrm{d}^{2} P / \mathrm{d} X^{2}$ also remains negative because $\mathrm{d} P / \mathrm{d} X$ remains negative. For $X<c, \mathrm{~d} P / \mathrm{d} X(<0)$ increases, i.e., $|\mathrm{d} P / \mathrm{d} X|$ decreases in the $-X$ direction because $\mathrm{d}^{2} P / \mathrm{d} X^{2}<0$. When $\mathrm{d} P / \mathrm{d} X(<0)$ comes close to 0 in consequence of its increase in the $-X$ direction, $\mathrm{d}^{2} P / \mathrm{d} X^{2}(<0)$ also comes close to 0 , and hence, $\mathrm{d} P / \mathrm{d} X$ and $\mathrm{d}^{2} P / \mathrm{d} X^{2}$ cannot become positive even if $X \rightarrow-\infty$, and they simultaneously converge to 0 for $X \rightarrow-\infty$. Therefore, $\mathrm{d} P / \mathrm{d} X$ and $\mathrm{d}^{2} P / \mathrm{d} X^{2}$ are negative at any point in the lubricated region. Due to the above tendencies of $\mathrm{d} P / \mathrm{d} X$ and $\mathrm{d}^{2} P / \mathrm{d} X^{2}$, the pressure distribution becomes convex upward and $P$ is asymptotic to its maximum for $X \rightarrow-\infty$ in the case of $D>1$. Let us check examples of the pressure distribution for $D>1$. Figure 11 shows pressure distributions for $D_{0}=13.186$, which corresponds to $h_{0}=1 \mu \mathrm{m}$ under atmospheric conditions with $p_{\mathrm{a}}$ and $T_{0}$. The bearing numbers $\Lambda=0,1.09643,10.9643,109.643$, and 1096.43 correspond to $U=0,0.1 \mathrm{~m} / \mathrm{s}, 1 \mathrm{~m} / \mathrm{s}, 10 \mathrm{~m} / \mathrm{s}$, and $100 \mathrm{~m} / \mathrm{s}$, respectively, under the condition of $l=10 \mathrm{~mm}$. All pressure distributions in Fig. 11 are convex upward. In particular, in the case of large $\Lambda$, the pressure is asymptotic to its maximum, i.e., $P_{\mathrm{i}}$ for $X \rightarrow 0$, and consequently, high average gas pressure is obtained.

Next let us consider the case of $D<1$, in which $\mathrm{d} Q_{\mathrm{P}}(D) / \mathrm{d} D$ is negative. Figure 12 shows pressure distributions for $D_{0}=0.013186$, which corresponds to $h_{0}=0.001 \mu \mathrm{m}$ under atmospheric conditions with $p_{\mathrm{a}}$ and $T_{0}$. The bearing numbers $0,1096.43,10964.3$, and 109643 correspond to $U=0,0.1 \mathrm{~m} / \mathrm{s}, 1 \mathrm{~m} / \mathrm{s}$, and $10 \mathrm{~m} / \mathrm{s}$, respectively, under the condition of $l=10 \mu \mathrm{m}$. In the case of $\Lambda=0$, the value in the square brackets in Eq. (21) becomes negative at the point $X=c$, where $\mathrm{d} P / \mathrm{d} X<0$, because $\mathrm{d} Q_{\mathrm{P}}(D) / \mathrm{d} D<0$ for $D<1$, and hence, $\mathrm{d}^{2} P / \mathrm{d} X^{2}$ becomes positive. For $X<c, \mathrm{~d} P / \mathrm{d} X(<0)$ decreases, i.e., $|\mathrm{d} P / \mathrm{d} X|$ increases in the $-X$ direction because $\mathrm{d}^{2} P / \mathrm{d} X^{2}>0$. Since $\mathrm{d} P / \mathrm{d} X$ remains negative, $\mathrm{d}^{2} P / \mathrm{d} X^{2}$ remains positive. For $X>c, \mathrm{~d} P / \mathrm{d} X(<0)$ increases, i.e., $|\mathrm{d} P / \mathrm{d} X|$ decreases in the $X$ direction because $\mathrm{d}^{2} P / \mathrm{d} X^{2}>0$, and hence, $\mathrm{d}^{2} P / \mathrm{d} X^{2}$ decreases, but remains positive because $\mathrm{d} P / \mathrm{d} X$ cannot become positive. When $\mathrm{d} P / \mathrm{d} X$ tries to change from negative to positive, $\mathrm{d}^{2} P / \mathrm{d} X^{2}$ becomes 0 , and hence, $\mathrm{d} P / \mathrm{d} X$ cannot change its sign. Therefore, the pressure distribution for the case of $\Lambda=0$ and $D<1$ is convex downward since $\mathrm{d}^{2} P / \mathrm{d} X^{2}$ is positive at any point in the lubricated region. The pressure distribution for $\Lambda=0$ in Fig. 12 is slightly convex downward.

If $\Lambda$ becomes larger than a certain value, the value in the square brackets in Eq. (21) will change from negative to positive. In the case of large $\Lambda$, the pressure distribution becomes convex upward because $\mathrm{d}^{2} P / \mathrm{d} X^{2}$ becomes negative due to the positive value in the square brackets and the negative gradient $\mathrm{d} P / \mathrm{d} X$, and the pressure is asymptotic to its maximum $P_{\mathrm{i}}$ for $X \rightarrow 0$ as shown in Fig. 12 in the same manner as in the aforementioned case of $D>1$. Therefore, high average gas pressure is obtained in the case of large $\Lambda$. Since the pressure 
gradient becomes very steep in the neighborhood of the outlet in the case of large $\Lambda$, it is possible that the negative value of the first term in the square brackets may become superior to the positive value of the second term, i.e., the value in the brackets may change from positive to negative, and then it is possible that $\mathrm{d}^{2} P / \mathrm{d} X^{2}$ may become positive in the neighborhood of the outlet. Although the positive $\mathrm{d}^{2} P / \mathrm{d} X^{2}$ will make the pressure distribution convex downward, it may appear only in the neighborhood of the outlet for large $\Lambda$. Even in such a case, a high average gas pressure will be obtained since the pressure gradient is very steep in the neighborhood of the outlet and the pressure in most regions except the neighborhood of the outlet is close to its maximum $P_{\mathrm{i}}$.

Note that the situations of the pressure distributions between two parallel sliding plates shown in Figs. 7 and 10 are more complicated. In those cases, not only the bearing number $\Lambda$ but also the characteristic inverse Knudsen number $D_{0}$ changes with changing $h_{0}$. In order to understand the mixed effect of two parameters $\Lambda$ and $D_{0}$, let us return to the discussion of Eq. (18). Equation (18) can be non-dimensionalized by using the non-dimensional quantities given by Eq. (19) as follows:

$$
\left[Q_{\mathrm{P}}(D)\left(-\frac{\mathrm{d} P}{\mathrm{~d} X}\right)\right]_{\mathrm{o}}-\left[Q_{\mathrm{P}}(D)\left(-\frac{\mathrm{d} P}{\mathrm{~d} X}\right)\right]_{\mathrm{i}}=\frac{\Lambda D_{0}}{6}\left(P_{\mathrm{i}}-P_{\mathrm{o}}\right) .
$$

This equation indicates what induces a convex upward pressure distribution, i.e., feature (2) of pressure distributions. As well as the case when $h_{0}$ decreases in the RHS of Eq. (18), the increase of $\Lambda D_{0}$ increases the RHS of Eq. (22) and induces a gentler pressure gradient $(-\mathrm{d} P / \mathrm{d} X)_{\mathrm{i}}$ at the inlet and a steeper pressure gradient $(-\mathrm{d} P / \mathrm{d} X)_{\mathrm{o}}$ at the outlet. Therefore, feature (2) becomes stronger as $\Lambda$ increases under constant $D_{0}$ in Figs. 11 and 12. Furthermore, feature (2) also becomes stronger as $D_{0}$ increases under constant $\Lambda$. (Compare the pressure distributions for $\Lambda=1096.43$ in Figs. 11,12 ). As the clearance $h_{0}$ decreases, $\Lambda D_{0}$ increases in inverse proportion to $h_{0}$ because $\Lambda \propto h_{0}^{-2}$ and $D_{0} \propto h_{0}$. This is the reason why feature (2) becomes stronger as $h_{0}$ decreases in Figs. 7 and 10. On the other hand, in the case where the sliding speed $U$ is increased, $D_{0}$ is unchanged, while $\Lambda$ increases in proportion to $U$. Therefore, feature (2) of pressure distributions becomes stronger because of the increase of $\Lambda$, i.e., the increase of $\Lambda D_{0}$, as shown in Fig. 5 .

The above discussion of Eq. (22) suggests that the parameter $A D_{0}$ may determine the shape of pressure distributions over the flat region. However, we did not consider the change of $Q_{\mathrm{P}}(D)$ against $D$ in this discussion in the last paragraph as we did not do so when we discussed the effect of $h_{0}$ in Eq. (18) above. Although $Q_{\mathrm{P}}(D)$ varies slowly between 1.539 and 3.060 depending on $D$ for the range $0.01<D<10$ considered here, the change of $Q_{\mathrm{P}}(D)$ against $D$ is not negligible. Figure 13 shows pressure distributions for several inverse Knudsen numbers $D_{0}$ under the condition that $\Lambda D_{0}$ is fixed at 144.575. The values of $\Lambda$ are 10964.3, 1096.43, 109.643, and 10.9643 for $D_{0}=0.013186,0.13186,1.3186$, and 13.186 , respectively. The pressure distributions for different $D_{0}$ 's are similar under constant $\Lambda D_{0}$ although $\Lambda$ differs by a factor of up to a 1000 . But they do not coincide with one another. The reason for the difference can be understood from the difference of the magnitudes of $Q_{\mathrm{P}}\left(D_{0}\right)$. The flow rate coefficient $Q_{\mathrm{P}}\left(D_{0}\right)$ is $2.932,1.934,1.547$, and 3.282 for $D_{0}=0.013186,0.13186,1.3186$, and 13.186 , respectively. Since $Q_{\mathrm{P}}(D)$ is not so different from $Q_{\mathrm{P}}\left(D_{0}\right)$ over the parallel lubricated region, we consider $Q_{\mathrm{P}}(D)$ 's at the inlet and outlet appearing in Eq. (22) to be equal to $Q_{\mathrm{P}}\left(D_{0}\right)$ here. Consequently, it is found from Eq. (22) that smaller $Q_{\mathrm{P}}\left(D_{0}\right)$ makes the pressure gradient at the outlet steeper under the condition of fixed $A D_{0}$, i.e., under the condition of a fixed value of the RHS of Eq. (22). Therefore, feature (2) of a pressure distribution becomes stronger for smaller $Q_{\mathrm{P}}\left(D_{0}\right)$. We can say that in the range $0.01<D_{0}<10$, i.e., in the high Knudsen number flow regime $0.1<K n_{0}<100$, the parameter $\Lambda D_{0}$ plays a key role in determining of the shape of the pressure distribution over the flat region and the magnitude of inverse Knudsen number $D_{0}$ has a secondary effect which modifies the pressure distribution through $Q_{\mathrm{P}}\left(D_{0}\right)$.

So far, feature (2) in the high Knudsen number flow regime of $0.1<K n_{0}<100$ has been discussed. Now, the case of the continuum gas flow regime of $K n_{0} \ll 1$, i.e., $1 \ll D_{0}$, should be referred to for comparison. In the case of continuum gas flow, the flow rate coefficient is given by $Q_{\mathrm{P}}(D)=D / 6=D_{0} P H / 6$, and then, Eq. (22) results in

$\left[P\left(-\frac{\mathrm{d} P}{\mathrm{~d} X}\right)\right]_{\mathrm{o}}-\left[P\left(-\frac{\mathrm{d} P}{\mathrm{~d} X}\right)\right]_{\mathrm{i}}=\Lambda\left(P_{\mathrm{i}}-P_{\mathrm{o}}\right)$,

for the problem of two parallel plates, i.e., $H=1$. Eq. (20) also results in

$\frac{\mathrm{d}}{\mathrm{d} X}\left[P H^{3} \frac{\mathrm{d} P}{\mathrm{~d} X}-\Lambda P H\right]=0$.

The above two equations indicate that only the bearing number $\Lambda$ governs pressure distributions in the case of continuum gas flow regime, as is well known [24]. It is noteworthy that the governing factor of pressure distributions in a lubricated film differs between the continuum gas flow regime and the high Knudsen number gas flow regime. The former is $\Lambda$, whereas the latter is $\Lambda D_{0}$.

\subsection{Application of Obtained Knowledge to Design the Surface Texture}

In the previous subsections, the mechanism of high gas pressure generation on a textured surface was explained. Let us make good use of the knowledge obtained in the previous subsections to design the texture. 


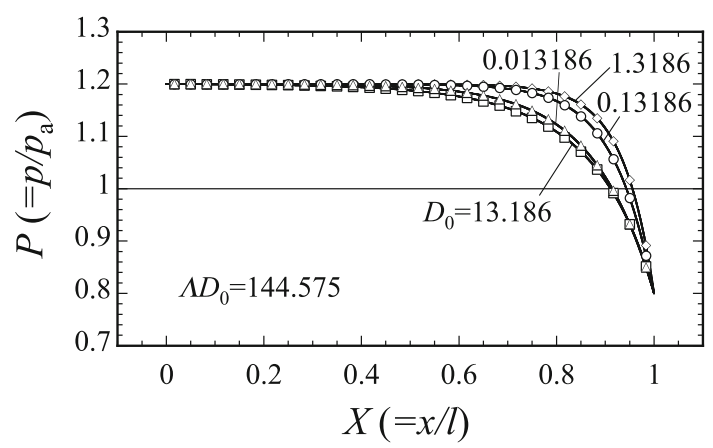

Fig. 13 Effect of the magnitude of the inverse Knudsen number $D_{0}$ under constant $A D_{0}$ on the pressure distribution in the case of the sliding of two parallel plates. $\left(P_{\mathrm{i}}=1.2, P_{\mathrm{o}}=0.8\right)$

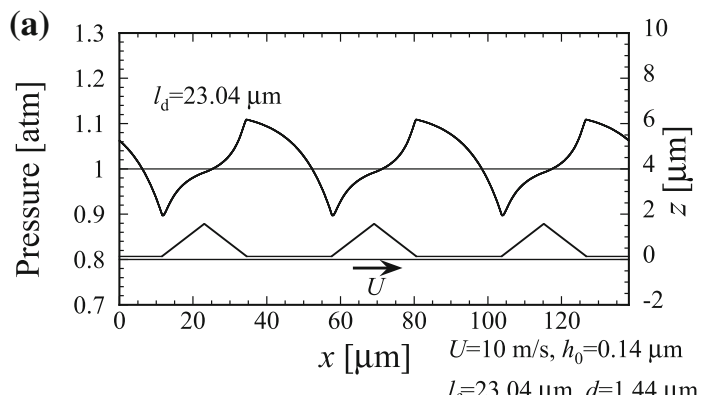

(b)

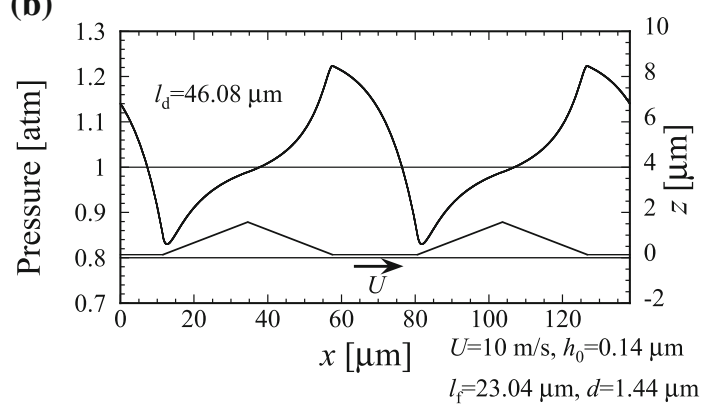

Fig. 14 Effect of the length $l_{\mathrm{d}}$ of the dimple region on the pressure distribution: a $l_{\mathrm{d}}=23.04 \mu \mathrm{m} ; \quad$ b $\quad l_{\mathrm{d}}=46.08 \mu \mathrm{m} . \quad\left(l_{\mathrm{f}}=23.04 \mu \mathrm{m}\right.$, $d=1.44 \mu \mathrm{m}, U=10 \mathrm{~m} / \mathrm{s}$, and $h_{0}=0.14 \mu \mathrm{m}$ )

Considering feature (1), the large pressure difference induced by a dimple is preferable to obtain high average gas pressure over the whole lubricated region in the case where feature (2) is also satisfied. The pressure difference for $h_{0}=0$ can be obtained by integrating $\mathrm{d} p / \mathrm{d} x$ in Eq. (15) over the dimple length $l_{\mathrm{d}}$. This indicates that a longer dimple with the same depth can induce a larger pressure difference. Let us check the effect of the length of the dimple region on the pressure distribution in the problem of a sliding surface with dimples. Figure 14 shows pressure distributions for a shorter dimple of $l_{\mathrm{d}}=23.04 \mu \mathrm{m}$ and a longer dimple of $l_{\mathrm{d}}=46.08 \mu \mathrm{m}$ for $h_{0}=0.14 \mu \mathrm{m}$ under the condition of $l_{\mathrm{f}}=23.04 \mu \mathrm{m}, d=1.44 \mu \mathrm{m}$, and $U=10 \mu / \mathrm{s}$. The pressure distribution for a short dimple shown in Fig. 14a is the same as the MGL result for $U=10 \mathrm{~m} / \mathrm{s}$ shown in Fig. 5. Let us refer to this case as the reference case. A larger pressure difference is obtained for the longer dimple as expected. The superficially averaged gas pressure $\bar{p}_{\text {surf }}$ for the reference case is $1.0190 \mathrm{~atm}$, whereas that for the case of the longer dimple is $1.0323 \mathrm{~atm}$. We can say that a higher averaged gas pressure can be obtained by extending the dimple length $l_{\mathrm{d}}$.

As mentioned in Sect. 3.4, the increase of $\Lambda D_{0}$ will result in the benefit of feature (2) of a pressure distribution over the flat region in the high Knudsen number flow regime. To increase $\Lambda D_{0}$ under the condition of constant values of the clearance $h_{0}$, the sliding speed $U$ and the ambient pressure $p_{\mathrm{a}}$, we can extend the length of the flat region. Let us double the length of parallel plates under the condition of $h_{0}=0.01 \mu \mathrm{m}, p_{\mathrm{i}}=1.2 \mathrm{~atm}, p_{\mathrm{o}}=0.8 \mathrm{~atm}$, and $U=10 \mathrm{~m} / \mathrm{s}$ in the problem of sliding of two parallel plates, as shown in Fig. 15. The pressure distribution for the case of the original length $l=10 \mu \mathrm{m}$ before extension is shown in Fig. 10 above. In this case, the pressure gradient at the inlet is 0 and that at the outlet is steep. If the plates are extended without changing the pressures $p_{\mathrm{i}}$ and $p_{\mathrm{o}}$ at the inlet and the outlet as shown in Fig. 15, the pressure $p$ will become equal to the maximum pressure $p_{\mathrm{i}}$ in the extended region $-l<x<0$, and hence, the pressure gradient $\mathrm{d} p / \mathrm{d} x$ will become 0 in this region, and furthermore, the pressure distribution in the original sliding region $0<x<l$ will be unchanged. This expected uniform pressure distribution in the extended region $-l<x<0$ indicates that there will be no component of the Poiseuille flow and that the flow rate of the Couette flow component will be constant in this region. Consequently, this expected distribution satisfies the continuity equation in the extended region $-l<x<0$. Figure 16 shows pressure distributions obtained by MGL calculations for the original plates and for the extended plates. Such pressure distributions as expected above have been obtained. The pressure distributions over the original sliding region $0<x<l$ for both cases agree exactly. This is because $\mathrm{d} p / \mathrm{d} x=0$ at $x=0$ in both cases.

Let us check the effect of extending the length of the flat region on the pressure distribution in the problem of a sliding surface with dimples. Figures 17 and 18 show pressure distributions for the shorter original flat region of $l_{\mathrm{f}}=23.04 \mu \mathrm{m}$ and the longer extended flat region of $l_{\mathrm{f}}=46.08 \mu \mathrm{m}$ for the cases of narrower clearance $h_{0}=0.0028 \mu \mathrm{m}$ and wider clearance $h_{0}=0.14 \mu \mathrm{m}$, respectively, under the condition of $l_{\mathrm{d}}=23.04 \mu \mathrm{m}$, $d=1.44 \mu \mathrm{m}$, and $U=10 \mathrm{~m} / \mathrm{s}$. In the case of $h_{0}=0.0028 \mu \mathrm{m}$, the gradient at the inlet of the flat region is 0 even for the shorter original flat region. Therefore, as well as the case of Fig. 16, the pressure distribution in the right side of the longer flat region in Fig. 17b agrees well with that in the original flat region in Fig. 17a, and the 


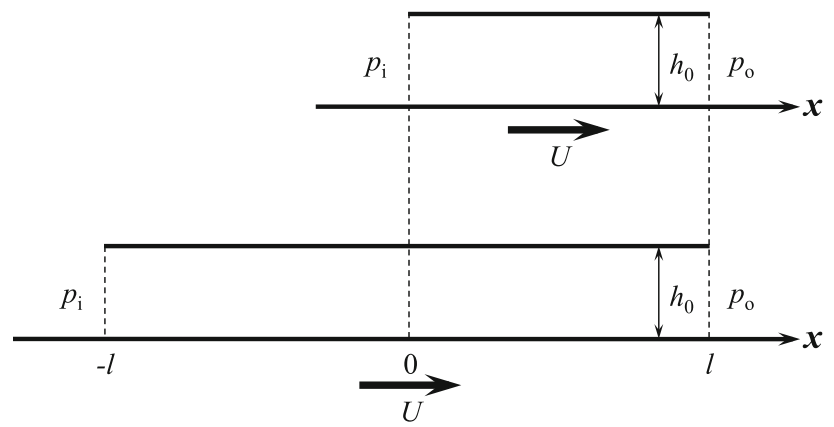

Fig. 15 Extension of the length of the sliding parallel plates

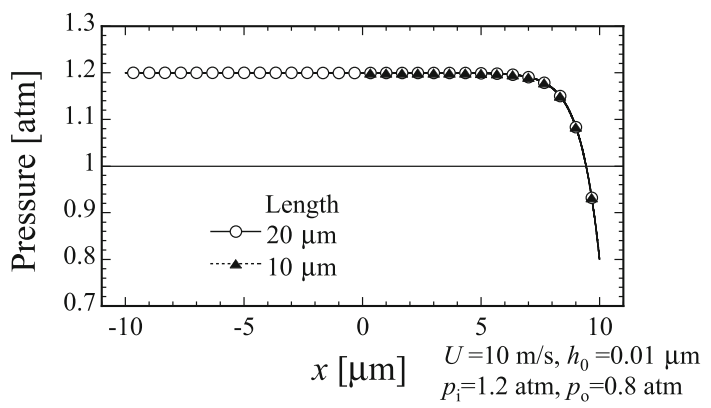

Fig. 16 Effect of the length of the sliding parallel plates on the pressure distribution. ( $h_{0}=0.01 \mu \mathrm{m}, p_{\mathrm{i}}=1.2 \mathrm{~atm}, p_{\mathrm{o}}=0.8 \mathrm{~atm}$, and $U=10 \mathrm{~m} / \mathrm{s}$ )

pressure is uniform and equal to its maximum over the leftside extended part of the longer flat region in Fig. 17b. In the case of $h_{0}=0.0028 \mu \mathrm{m}$, the superficially averaged gas pressure $\bar{p}_{\text {surf }}$ for the case of the shorter original flat region is $1.3452 \mathrm{~atm}$, whereas that for the case of the longer extended flat region is $1.4597 \mathrm{~atm}$. In the case of the wider clearance $h_{0}=0.14 \mu \mathrm{m}$, feature (2) of a pressure distribution becomes stronger by extending the flat region, as shown in Fig. 18. This is because the value $\Lambda D_{0}$ for the flat region increases in proportion to its length $l_{\mathrm{f}}$. In the case of $h_{0}=0.14 \mu \mathrm{m}$, the superficially averaged gas pressure $\bar{p}_{\text {surf }}$ for the case of the shorter original flat region is $1.0190 \mathrm{~atm}$, whereas that for the case of the longer extended flat region is $1.0325 \mathrm{~atm}$. We can say that the higher averaged gas pressure $\bar{p}_{\text {surf }}$ can be obtained by extending the length of the flat region, $l_{\mathrm{f}}$.

The results obtained above indicate that if we extend the length of both regions, we will obtain higher average gas pressure. Figure 19 shows the pressure distribution for the case where the lengths of both regions for the reference case shown in Fig. 14a are doubled. The superficially averaged gas pressure $\bar{p}_{\text {surf }}$ increases from 1.0190 to $1.0601 \mathrm{~atm}$ by doubling both lengths. Extending the whole surface structure doubly in the sliding direction without changing its depth results in doubling the bearing number $\Lambda\left(=6 \mu U l_{\mathrm{T}} / p_{\mathrm{a}} h_{0}^{2}\right)$ without changing the characteristic
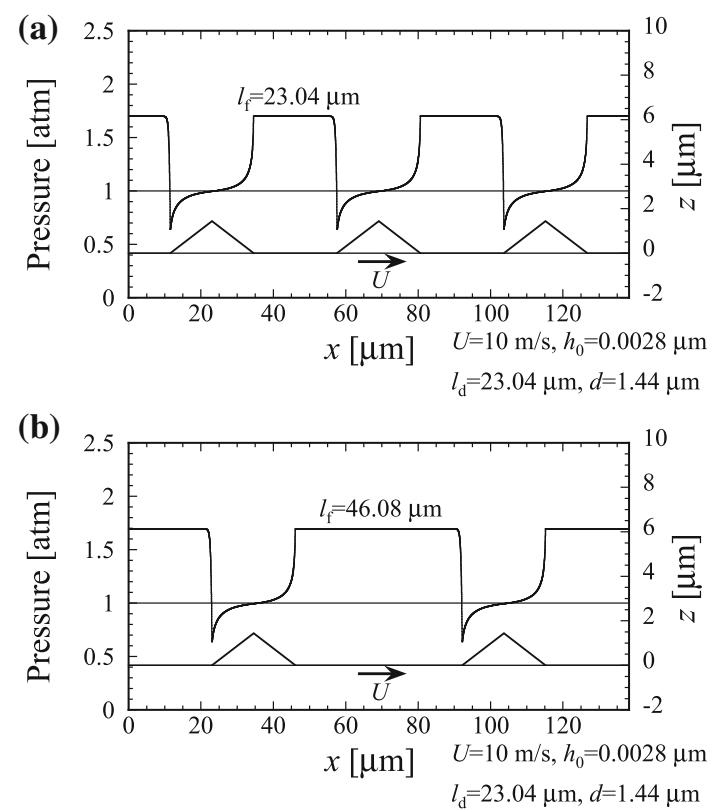

Fig. 17 Effect of the length $l_{\mathrm{f}}$ of the flat region on the pressure distribution: a $l_{\mathrm{f}}=23.04 \mu \mathrm{m} ; \quad$ b $\quad l_{\mathrm{f}}=46.08 \mu \mathrm{m} . \quad\left(l_{\mathrm{d}}=23.04 \mu \mathrm{m}\right.$, $d=1.44 \mu \mathrm{m}, U=10 \mathrm{~m} / \mathrm{s}$, and $h_{0}=0.0028 \mu \mathrm{m}$ )
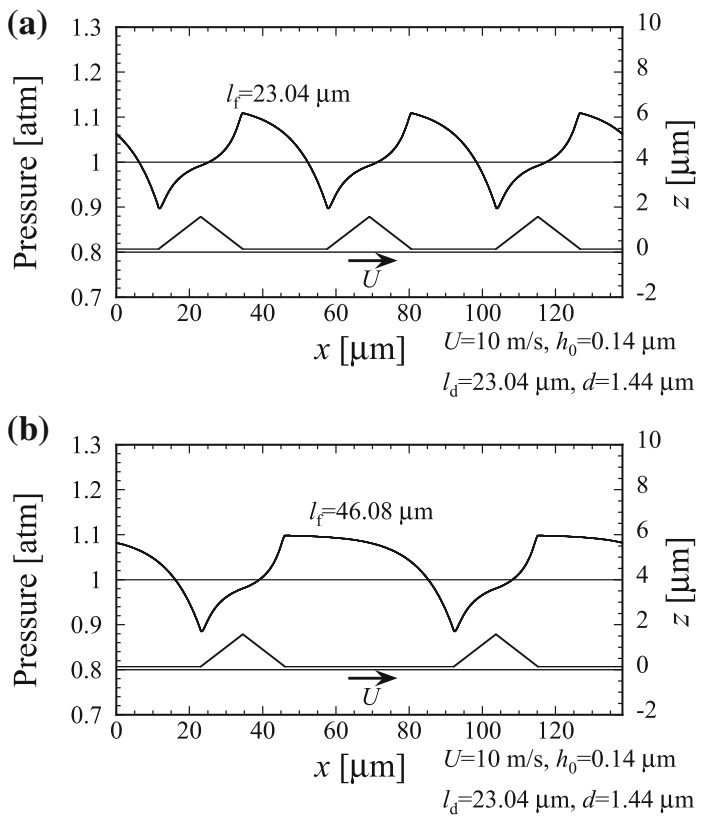

Fig. 18 Effect of the length $l_{\mathrm{f}}$ of the flat region on the pressure distribution: a $l_{\mathrm{f}}=23.04 \mu \mathrm{m} ; \quad$ b $\quad l_{\mathrm{f}}=46.08 \mu \mathrm{m} . \quad\left(l_{\mathrm{d}}=23.04 \mu \mathrm{m}\right.$, $d=1.44 \mu \mathrm{m}, U=10 \mathrm{~m} / \mathrm{s}$, and $\left.h_{0}=0.14 \mu \mathrm{m}\right)$

inverse Knudsen number $D_{0}$ in the non-dimensional MGL equation (20). From the definition of $\Lambda$, doubling the sliding speed $U$ also results in doubling the bearing number $\Lambda$ without changing $D_{0}$. Therefore, we can say that both operations have the same effect on the pressure distribution. The same non-dimensional pressure distribution will 


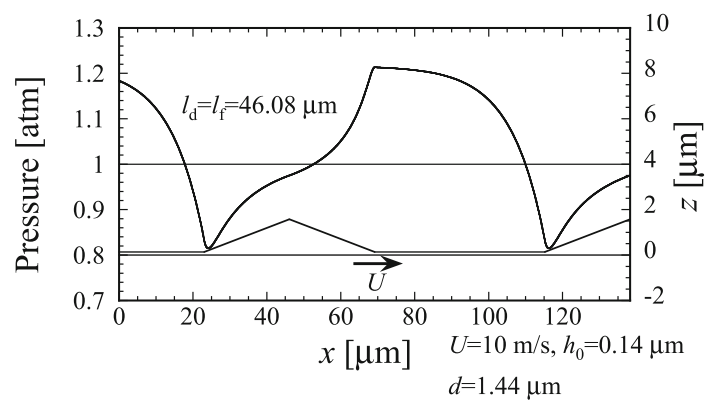

Fig. 19 Pressure distribution for the case where the lengths of both the dimple region and the flat region for the reference case shown in Fig. $14 \mathrm{a}$ are doubled. $\left(l_{\mathrm{d}}=l_{\mathrm{f}}=46.08 \mu \mathrm{m}, d=1.44 \mu \mathrm{m}, U=10 \mathrm{~m} / \mathrm{s}\right.$, and $\left.h_{0}=0.14 \mu \mathrm{m}\right)$

be obtained over the non-dimensionalized space as a result of either operation. If the whole surface structure is extended in the sliding direction without changing its depth, the high average gas pressure $\bar{p}_{\text {surf }}$ can be obtained as if the sliding speed $U$ is increased at the same rate. Conversely, if we double the length of the whole surface structure in the sliding direction without changing its depth, the same average gas pressure $\bar{p}_{\text {surf }}$ can be obtained even when half sliding speed $U$ is used.

\subsection{Optimal Choice of the Dimple Location}

So far, pressure distributions on a textured surface have been discussed under the condition that the spatially averaged pressure $\bar{p}_{\text {space }}$ is equal to the ambient pressure $p_{\mathrm{a}}$. In the case where the textured surface pattern is repeated infinitely in the sliding direction as the circumferential gas seal with dimples in [4], applying such a condition would be reasonable. However, in the case of the textured slider with finite size such as a typical magnetic recording slider, the lubricated region is connected to ambient air at both ends of the slider. The pressures at both ends of the textured slider should be assumed to be the ambient pressure $p_{\mathrm{a}}$. The average gas pressure exerted on the textured slider surface will depend on which part of the repeated surface pattern is connected to ambient air. Settling the location of the boundaries with ambient air in the repeated surface pattern corresponds to settling the location of the dimple in the period of the repeated surface pattern. See Fig. 20. The dashed lines in Fig. 20 represent borders between two adjacent periods of the repeated surface pattern. These figures show where the dimple is located within the period, i.e., they also show which part of the repeated surface pattern is connected to ambient air. In this subsection, the effect of the dimple location, i.e., the boundary location in the repeated surface pattern, on obtainment of high average gas pressure is discussed.

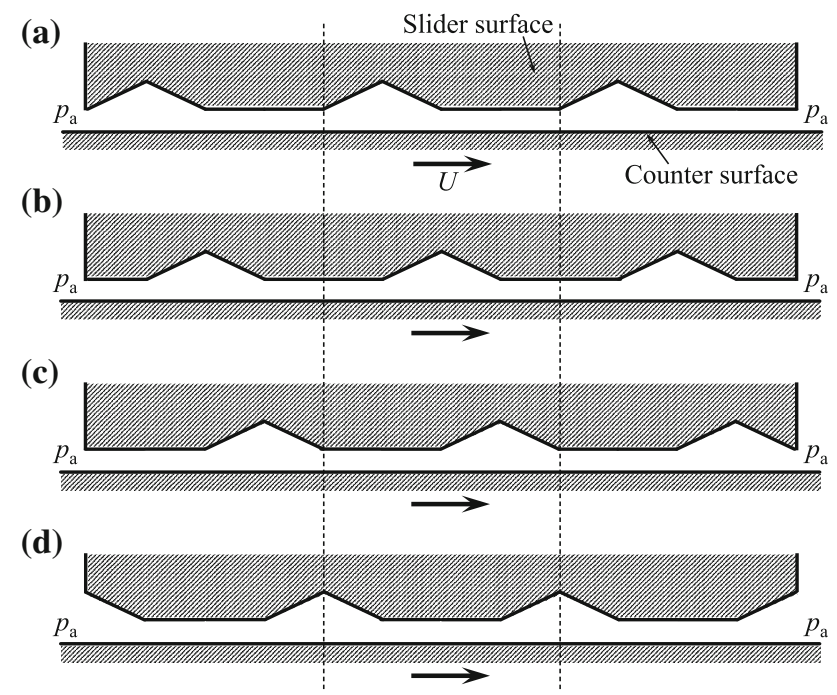

Fig. 20 Dimple locations in the period of the repeated surface pattern: a left side; $\mathbf{b}$ middle; $\mathbf{c}$ right side; $\mathbf{d}$ both ends

Figure 20 shows the four dimple locations considered here. At the beginning and the end of the repeated surface pattern, the lubricated region is connected to ambient air with $p_{\mathrm{a}}$. Figure 21 shows pressure distributions for these dimple locations. The line figure in the lower half of each figure shows the shape of the lubricated region. See also the pressure distribution shown in Fig. 14a, which is the corresponding case where the conditions except the presence or absence of boundaries with ambient air are the same as in the cases shown in Fig. 21. The pressure distributions in Figs. 14a and 21 have almost the same shape. Depending on the values of the pressure at the points in Fig. 14a which correspond to the boundaries with ambient air in Fig. 20, the pressure distribution is shifted upward or downward as shown in Fig. 21. If the points with lower pressure in Fig. 14a are chosen as the boundaries with ambient air, the pressure distribution is shifted upward. Conversely, if the points with higher pressure are chosen, the pressure distribution is shifted downward.

Undoubtedly, choosing the points where the gas pressure is minimum in the pressure distribution of Fig. 14a as the boundaries with ambient air is the best policy to obtain high gas pressure. The minimum points are located in the vicinities of the inlets of the dimples in the cases considered here, as shown in Figs. 7, 14, 19, and so on. Therefore, the best choice of the boundary location is "the inlet of the dimple," and in the other words, the best choice of the dimple location is "the left side of the period," as shown in Fig. 20a. If the inlets of the dimples are chosen as the boundaries, the pressures at all points except the vicinities of the inlets of the dimples are higher than the ambient pressure $p_{\mathrm{a}}$, as shown in Fig. 21a. Since the minimum point of the gas 

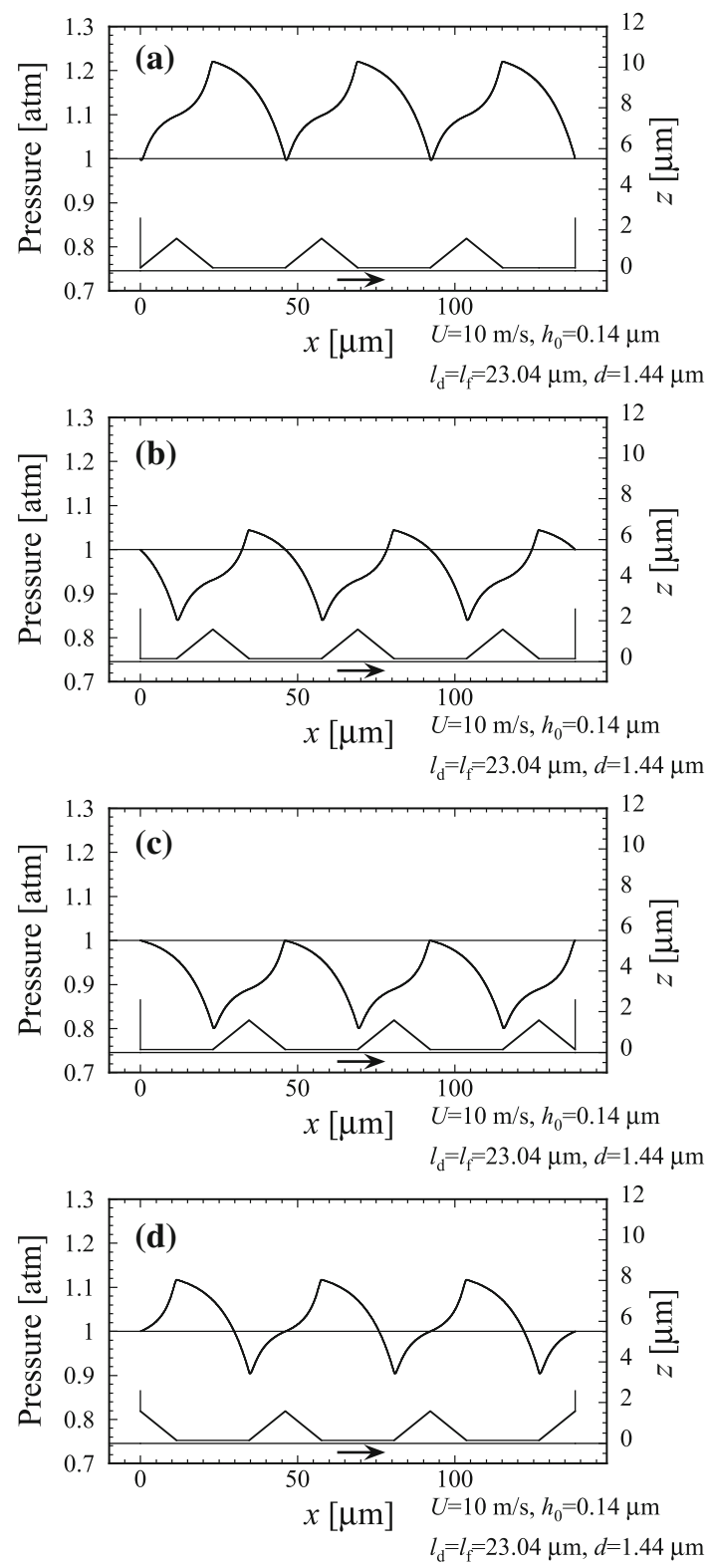

Fig. 21 Pressure distributions for the four dimple locations: a left side; b middle; c right side; d both ends. $\left(l_{\mathrm{d}}=l_{\mathrm{f}}=23.04 \mu \mathrm{m}\right.$, $d=1.44 \mu \mathrm{m}, U=10 \mathrm{~m} / \mathrm{s}$, and $h_{0}=0.14 \mu \mathrm{m}$ )

pressure is slightly separated from the inlet of the dimple, as shown in Figs. 7, 14, 19, and so on, the gas pressure only in the very small regions near the inlets of the dimples is less than $p_{\mathrm{a}}$ in Fig. 21a.

Putting the dimple at both ends of the period, as shown in Fig. 20d, i.e., choosing the centers of the dimples as the boundaries with ambient air, is acceptable, as shown in Fig. 21d. The pressure distribution in Fig. 21d is close to that in Fig. 14a for the case where the spatially averaged gas pressure $\bar{p}_{\text {space }}$ is set at the ambient pressure $p_{\mathrm{a}}$. The reason for this is as follows. Since the space around the center of the dimple occupies the majority of the whole volume of one period of the lubricated region, the pressure at the center of the dimple becomes close to $\bar{p}_{\text {space }}$, and hence, close to $p_{\mathrm{a}}$ in Fig. 14a for the case where $\bar{p}_{\text {space }}=p_{\mathrm{a}}$. Therefore, the two pressure distributions in Figs. 21d and 14a are close.

Putting the dimple in the middle or in the right side of the period, as shown in Fig. $20 \mathrm{~b}$ or c, i.e., choosing the middle points or the inlets of the flat regions as the boundaries with ambient air, is unsuitable for obtaining high gas pressure. The pressure becomes rather low for such boundary locations, as shown in Fig. 21b, c. This is because the pressure in most of the flat region except the vicinity of its outlet is high, compared with that at the other part, especially in the case of small clearance, as shown in Fig. 17. Such a choice of boundary location makes the pressure in most of the lubricated region lower than the ambient pressure $p_{\mathrm{a}}$, as shown in Fig. 21b, c.

As we mentioned above, the gas pressure monotonically increases over the dimple region in the $x$ direction, i.e, in the direction of the counter surface's motion. The other side of the coin is that the gas pressure monotonically decreases over the dimple region in the $-x$ direction, i.e., in the direction opposite to the counter surface's motion. In the case where the dimple's inlet is connected to the point where the pressure is as high as the ambient pressure $p_{\mathrm{a}}$, as shown in Fig. 21a, the pressure difference between the inlet and the outlet of the dimple plays a role in making the average gas pressure $\bar{p}_{\text {surf }}$ higher. Remember that the left edge and the right edge of the dimple were termed "inlet" and "outlet", respectively, with respect to the direction of net gas flow, i.e., with respect to the direction of the counter surface's motion in Sect. 3.1. Conversely, in the case where the dimple's outlet is connected to the point where the pressure is as high as the ambient pressure $p_{\mathrm{a}}$, as shown in Fig. 21b, c, the pressure difference between the two ends of the dimple plays a role in lowering the average gas pressure $\bar{p}_{\text {surf }}$. The gas pressures $\bar{p}_{\text {surf }}$ averaged over the whole surface for the cases of Fig. 21a-d are 1.1256, $0.9575,0.9153$, and $1.0268 \mathrm{~atm}$, respectively. It can be concluded that the proper setting of the dimple location is important to obtain high average gas pressure.

Figure 22 shows the effect of the minimum clearance $h_{0}$ on the gas pressure $\bar{p}_{\text {surf }}$ averaged over the whole bottom surface of the slider for the four dimple locations defined in Fig. 20. In the cases where the dimple is located in the "left side" or located at "both ends" of the period as shown in Fig. $20 \mathrm{a}$, d, the gas pressure $\bar{p}_{\text {surf }}$ increases from the ambient pressure $p_{\mathrm{a}}(=1 \mathrm{~atm})$ as the clearance $h_{0}$ decreases. Therefore, a higher lift force $\bar{p}_{\text {surf }}-p_{\mathrm{a}}$ can be obtained for smaller $h_{0}$ for such dimple locations. In contrast, in the cases where the dimple is located in the "middle" or the "right side" of the period, as shown in Fig. 20b, c, the gas 


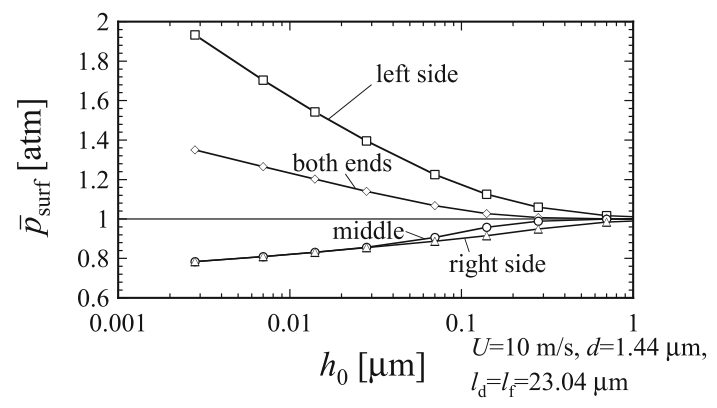

Fig. 22 Effect of the clearance $h_{0}$ on superficially averaged gas pressure $\bar{p}_{\text {surf }}$ for the four dimple locations defined in Fig. 20. $\left(l_{\mathrm{d}}=l_{\mathrm{f}}=23.04 \mu \mathrm{m}, d=1.44 \mu \mathrm{m}, U=10 \mathrm{~m} / \mathrm{s}\right)$

pressure $\bar{p}_{\text {surf }}$ decreases from the ambient pressure $p_{\mathrm{a}}(=$ $1 \mathrm{~atm})$ as $h_{0}$ decreases. Since the superficially averaged gas pressure $\bar{p}_{\text {surf }}$ is less than $p_{\mathrm{a}}$ in the case of such dimple locations, the downforce $p_{\mathrm{a}}-\bar{p}_{\text {surf }}$ is exerted on the slider. Figure 22 shows that the downforce obtained for such dimple locations becomes stronger as $h_{0}$ decreases. Note that as $h_{0}$ decreases, the deviation of $\bar{p}_{\text {surf }}$ from $p_{\mathrm{a}}$ becomes larger for any dimple location. This is because the pressure difference between the two ends of the dimple, which induces lift force or downforce, becomes stronger as $h_{0}$ decreases. The gas pressures $\bar{p}_{\text {surf }}$ for the dimple location "both ends" shown in Fig. 22 are close to those for the case of $\bar{p}_{\text {space }}=p_{\mathrm{a}}$ shown in Fig. 8 for the aforementioned reason. The gas pressures $\bar{p}_{\text {surf }}$ for the optimal dimple location "left side" shown in Fig. 22 are much higher than those for the case of $\bar{p}_{\text {space }}=p_{\text {a }}$ shown in Fig. 8 .

The knowledge about pressure generation on a textured surface, which was obtained in the foregoing subsections under the condition of $\bar{p}_{\text {space }}=p_{\mathrm{a}}$, is applicable to the cases where the dimple is located in the "left side" or located at "both ends" of the period. Even in those cases, features (1) and (2) of a pressure distribution play a key role in the generation of higher gas pressure, and furthermore, smaller clearances, longer dimple regions, longer flat regions, and higher sliding speed $U$ can make the average gas pressure $\bar{p}_{\text {surf }}$ higher. However, note that a wrong choice of the dimple location in the period of the repeated surface pattern can spoil the desirable effects of features (1) and (2), as shown in Fig. $21 \mathrm{~b}$, c. In the cases of such dimple locations as "right side" and "middle" in Fig. 20b, c, feature (1), i.e., the pressure difference between the two ends of the dimple plays a role in reducing the average gas pressure $\bar{p}_{\text {surf }}$ as mentioned above.

Figure 23 shows the effects of the length $l_{\mathrm{f}}$ of the flat region and the length $l_{\mathrm{d}}$ of the dimple region on pressure generation in the case where the lubricated region is connected to ambient air at both ends of the textured slider and the dimple is located in the "left side" of the period of the
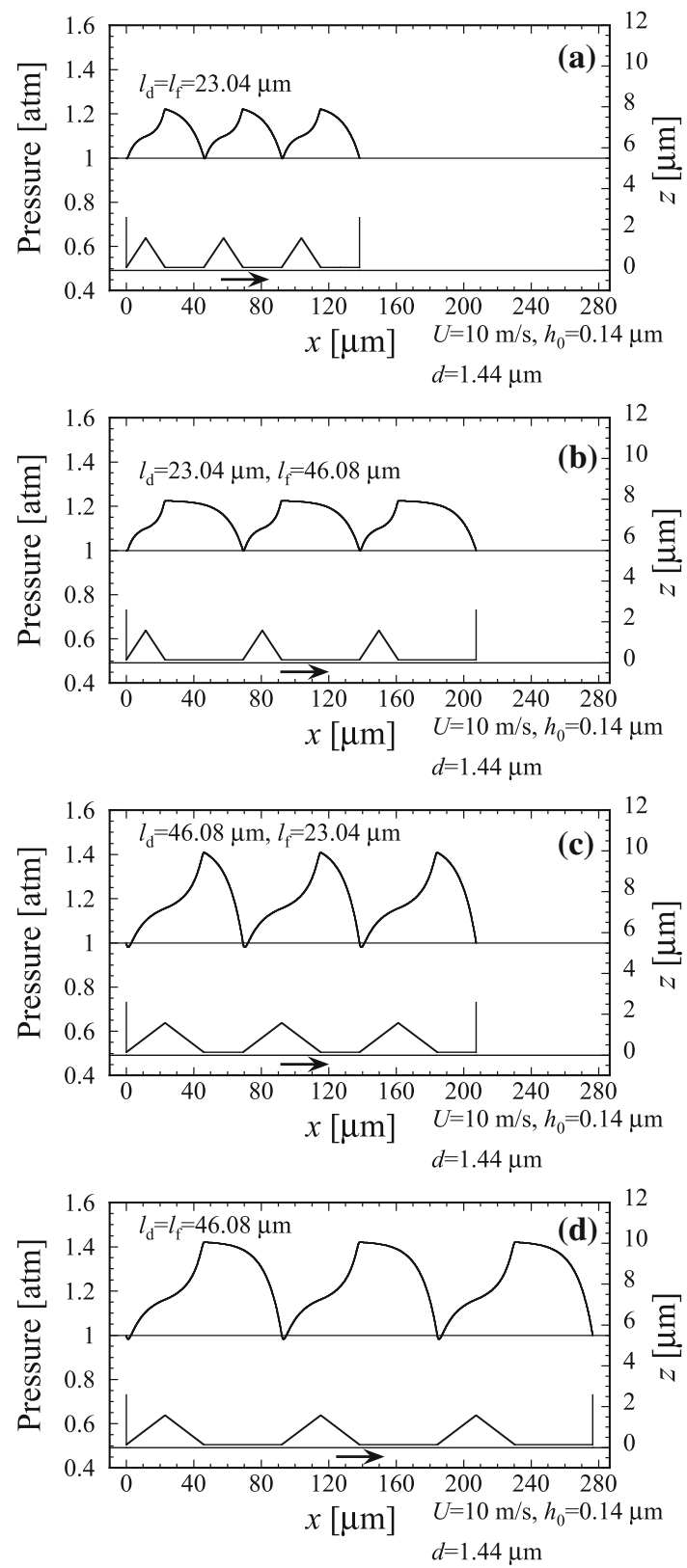

Fig. 23 Effects of the length $l_{\mathrm{f}}$ of the flat region and the length $l_{\mathrm{d}}$ of the dimple region on the pressure distribution in the case where the dimple is located in the left side of the period of the repeated surface pattern as shown in Fig. 20a: a $l_{\mathrm{d}}=l_{\mathrm{f}}=23.04 \mu \mathrm{m} ; \mathbf{b} l_{\mathrm{d}}=23.04 \mu \mathrm{m}$, $l_{\mathrm{f}}=46.08 \mu \mathrm{m} ; \mathbf{c} l_{\mathrm{d}}=46.08 \mu \mathrm{m}, l_{\mathrm{f}}=23.04 \mu \mathrm{m} ; \mathbf{d} l_{\mathrm{d}}=l_{\mathrm{f}}=46.08 \mu \mathrm{m}$. $\left(d=1.44 \mu \mathrm{m}, U=10 \mathrm{~m} / \mathrm{s}\right.$, and $\left.h_{0}=0.14 \mu \mathrm{m}\right)$

repeated surface pattern, as shown in Fig. 20a. Note that the case shown in Fig. 23a is the same as the case shown in Fig. $21 \mathrm{a}$. In the case where the length $l_{\mathrm{f}}$ of the flat region is doubled, as shown in Fig. $23 \mathrm{~b}$, the average gas pressure $\bar{p}_{\text {surf }}$ increases from $1.1256 \mathrm{~atm}$ for the case of Fig. 23a to $1.1538 \mathrm{~atm}$. In the case where the length $l_{\mathrm{d}}$ of the dimple region is doubled, as shown in Fig. 23c, the average gas pressure $\bar{p}_{\text {surf }}$ increases to $1.2011 \mathrm{~atm}$. Furthermore, in the 
case where the lengths of both regions are doubled, as shown in Fig. $23 \mathrm{~d}$, the average gas pressure $\bar{p}_{\text {surf }}$ increases to $1.2521 \mathrm{~atm}$. In the case where the sliding speed $U$ is doubled from the case of Fig. $23 \mathrm{a}, \bar{p}_{\text {surf }}$ increases from 1.1256 to $1.2521 \mathrm{~atm}$ (not shown), as well as in the case where the length of whole region is doubled as shown in Fig. 23d. This is because both operations have the same effect on the pressure distribution, as mentioned in Sect. 3.5.

As mentioned in Sect. 3.2, the asymmetric pressure distribution over the dimple region in which the pressure rise over the converging region is greater than the pressure drop over the diverging region is beneficial for obtaining high average gas pressure in the case where $\bar{p}_{\text {space }}$ is set at $p_{\mathrm{a}}$. Note that the words "pressure rise" and "pressure drop" mean "rise" and "drop" with respect to the gas pressure averaged in the dimple region. In the case where the minimum points of gas pressure are chosen as the boundaries with ambient air, as shown in Fig. 21a, the aforementioned asymmetric shape of pressure distributions in the dimple region is no longer beneficial for obtaining high gas pressure. Rather, inducing a large pressure difference between the two ends of the dimple is important for realizing high gas pressure generation.

\section{Conclusions}

From our theoretical analysis, the following points can be concluded:

1. The following two features of a pressure distribution on a textured surface play a key role in generating high gas pressure. The first feature, namely "feature (1)," is that the gas pressure increases in the direction of the counter surface's motion over the dimple region. The second feature, namely "feature (2)," is that the pressure distribution over the flat region is convex upward. The combination of a gentler gradient at the inlet of the flat region and a steeper gradient at the outlet of the flat region is more beneficial because the high gas pressure obtained at the outlet of the dimple region can be maintained for a long distance in the flat region.

2. The causes of features (1) and (2) were explained theoretically on the basis of the continuity equation in the high Knudsen number flow regime and its derivative, i.e., the MGL equation.

3. In the continuum flow regime, the bearing number $\Lambda$ governs the pressure distribution, whereas in the high Knudsen number flow regime the product $\Lambda D_{0}$ plays a key role in determination of the pressure distribution over the flat region. The magnitude of inverse Knudsen number $D_{0}$ has a secondary effect which modifies the pressure distribution through the flow rate coefficient for the Poiseuille flow, $Q_{\mathrm{P}}\left(D_{0}\right)$. As $\Lambda D_{0}$ increases, feature (2) becomes stronger, i.e., the pressure gradient at the inlet of the flat region becomes gentler and that at the outlet of the flat region becomes steeper.

4. As the minimum clearance $h_{0}$ decreases or as the sliding speed $U$ increases, both features (1) and (2) become more remarkable. The effect of $h_{0}$ on feature (1) was explained by considering the situation where gas is leaking through the narrow clearance from the dimple to the next dimple in the case where both surfaces are almost in contact. As the clearance $h_{0}$ increases, the pressure increase over the dimple is reduced by increasing gas leakage. The reason why increasing $U$ enhances feature (1) can be understood from the fact that the pressure gradient $\mathrm{d} p / \mathrm{d} x$ in the dimple region is positive and is in proportion to $U$ in the case of $h_{0}=0$. The reason why decreasing $h_{0}$ and increasing $U$ enhances feature (2) is because both operations result in the increase of $\Lambda D_{0}$ since $\Lambda D_{0} \propto U / h_{0}$.

5. In the case where both ends of the textured slider with the repeated surface pattern are connected to ambient air, the choice of the dimple location in the period of the pattern is important. Putting the dimple in the inlet side of the period is optimal for generating high average gas pressure $\bar{p}_{\text {surf }}$, and hence, it produces high lift force. Putting the dimple at both ends of the period is acceptable, and it produces moderate lift force as much as that in the case where the spatially averaged gas pressure $\bar{p}_{\text {space }}$ is set at the ambient pressure $p_{\mathrm{a}}$. On the other hand, putting the dimple in the outlet side or in the middle of the period makes the superficially averaged gas pressure $\bar{p}_{\text {surf }}$ less than the ambient pressure $p_{\mathrm{a}}$, and hence, it produces downforce. Decreasing $h_{0}$ makes lift force and downforce stronger in each case due to enhancement of feature (1).

6. As expected on the basis of our understanding of the mechanism of high gas pressure generation, longer dimple regions, longer flat regions, and higher sliding speed increase the pressure $\bar{p}_{\text {surf }}$ in the case where the dimple is located in the inlet side of the period. If the whole surface structure is extended in the sliding direction without changing its depth, higher average gas pressure can be obtained as if the sliding speed $U$ is increased at the same rate, and conversely, the same pressure $\bar{p}_{\text {surf }}$ can be obtained even when the sliding speed $U$ is inversely reduced. For example, if the surface structure is doubled or tripled in the sliding direction without changing its depth, the same pressure $\bar{p}_{\text {surf }}$ can be obtained even when a sliding speed $U$ of one-half or one-third is used. 
Acknowledgments This work was supported by JSPS KAKENHI Grant Number 23560184. This work was also partly supported by the JSPS Core-to-Core Program, A. Advanced Research Networks, "International research core on smart layered materials and structures for energy saving". The DSMC simulations and the MGL calculations were carried out using the vector parallel supercomputer NEC SX-9 and scalar parallel supercomputer SGI AltixUV1000 of Advanced Fluid Information Research Center, Institute of Fluid Science, Tohoku University.

Open Access This article is distributed under the terms of the Creative Commons Attribution License which permits any use, distribution, and reproduction in any medium, provided the original author(s) and the source are credited.

\section{References}

1. Hamilton, D.B., Walowit, J.A., Allen, C.M.: A theory of lubrication by micro-irregularities. J. Basic Eng. Trans. ASME 88, 177-185 (1966)

2. Etsion, I., Burstein, L.: A model for mechanical seals with regular microsurface structure. Tribol. Trans. 39, 677-683 (1996)

3. Etsion, I., Kligerman, Y., Halperin, G.: Analytical and experimental investigation of laser-textured mechanical seal faces. Tribol. Trans. 42, 511-516 (1999)

4. Kligerman, Y., Etsion, I.: Analysis of the hydrodynamic effects in a surface textured circumferential gas seal. Tribol. Trans. 44, 472-478 (2001)

5. Wang, X., Kato, K., Adachi, K., Aizawa, K.: Loads carrying capacity map for the surface texture design of $\mathrm{SiC}$ thrust bearing sliding in water. Tribol. Int. 36, 189-197 (2003)

6. Zhou, L., Kato, K., Vurens, G., Talke, F.E.: The effect of slider surface texture on flyability and lubricant migration under near contact conditions. Tribol. Int. 36, 269-277 (2003)

7. Etsion, I.: State of the art in laser surface texturing. J. Tribol. Trans. ASME 127, 248-253 (2005)

8. Etsion, I.: Surface texturing. In: Bruce, R.W. (ed.) Handbook of Lubrication and Tribology, Volume II Theory and Design, 2nd edn, Chap. 53. CRC Press, Boca Raton (2012)

9. Raeymaekers, B., Etsion, I., Talke, F.E.: A model for magnetic tape/guide friction reduction by laser surface texturing. Tribol. Lett. 28, 9-17 (2007)

10. Murthy, A.N., Etsion, I., Talke, F.E.: Analysis of surface textured air bearing sliders with rarefaction effects. Tribol. Lett. 28 , 251-261 (2007)
11. Nakamori, I., Takagi, T., Takeno, T., Abe, T., Uchimoto, T., Kohama, Y.: Direct simulation of Monte Carlo analysis of nanofloating effect on diamond-coated surface. Diam. Relat. Mater. 14, 2122-2126 (2005)

12. Miki, H., Tsutsui, A., Takeno, T., Takagi, T.: Friction properties of partially polished CVD diamond films at different sliding speeds. Diam. Relat. Mater. 24, 167-170 (2012)

13. Takeno, T., Komoriya, T., Nakamori, I., Miki, H., Abe, T., Uchimoto, T., Takagi, T.: Tribological properties of partly polished diamond coatings. Diam. Relat. Mater. 14, 2118-2121 (2005)

14. Yonemura, S., Yamaguchi, M., Takeno, T., Miki, H., Takagi, T.: Effect of micro gas flow on low friction properties of diamond coating with partly polished surface. AIP Conf. Proc. 1084, 1153-1157 (2008)

15. Fukui, S., Kaneko, R.: Analysis of ultra-thin gas film lubrication based on linearized Boltzmann equation: first report-derivation of a generalized lubrication equation including thermal creep flow. J. Tribol. Trans. ASME 110, 253-261 (1988)

16. Alexander, F.J., Garcia, A.L., Alder, B.J.: Direct simulation Monte Carlo for thin-film bearings. Phys. Fluids 6, 3854-3860 (1994)

17. Huang, W., Bogy, D.B., Garcia, A.L.: Three-dimensional direct simulation Monte Carlo method for slider air bearings. Phys. Fluids 9, 1764-1769 (1997)

18. Bahukudumbi, P., Beskok, A.: A phenomenological lubrication model for the entire Knudsen regime. J. Micromech. Microeng. 13, 873-884 (2003)

19. Fukui, S., Yamane, K.: DSMC/MGL comparisons of stresses on slider air bearing with nanometer spacings. IEEE Trans. Magn. 38, 2153 (2002)

20. Bird, G.A.: Molecular Gas Dynamics and the Direct Simulation of Gas Flows. Oxford University Press, New York (1994)

21. Oran, E.S., Oh, C.K., Cybyk, B.Z.: Direct simulation Monte Carlo: recent advances and applications. Annu. Rev. Fluid Mech. 30, 403-441 (1998)

22. Nanbu, K.: Probability theory of electron-molecule, ion-molecule, molecule-molecule, and Coulomb collisions for particle modeling of materials processing plasmas and gases. IEEE Trans. Plasma Sci. 28, 971-990 (2000)

23. Fukui, S., Kaneko, R.: A database for interpolation of Poiseuille flow rates for high Knudsen number lubrication problems. J. Tribol. Trans. ASME 112, 78-83 (1990)

24. Andrés, L.S.: Compressible gas film lubrication. In: Bruce, R.W. (ed.) Handbook of Lubrication and Tribology, Volume II: Theory and Design, 2nd edn, Chap. 17. CRC Press, Boca Raton (2012) 